\title{
Statistical Mechanics of Vortices in an Inviscid Two-Dimensional Fluid
}

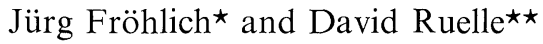 \\ IHES, F-91440 Bures-sur-Yvette, France
}

\begin{abstract}
In this paper we study rigorously the statistical mechanics of a gas of vortices in the thermodynamic limit. In this limit, no negative temperature states are found to exist.
\end{abstract}

\section{Introduction}

When the motion of a fluid may be considered as approximately two-dimensional, the fluid often exhibits well-defined vortices. The existence of such vortices is an experimental fact, which Poincaré already tried to explain ${ }^{1}$. If dissipation may be neglected, the motion of the vortices is Hamiltonian, and it is natural to study the "gas of vortices" by the methods of statistical mechanics. Onsager [20] has argued that when the total energy of the system is sufficiently large, the "gas of vortices" is in a "negative temperature state." He further argued that, in such a state, vortices of the same sign attract each other. In fact the coalescence of vortices of the same sign has later been observed in computer experiments (see Montgomery and Joyce [17]), and is claimed to explain in part the existence of large well-defined vortices ${ }^{2}$.

In this paper we study rigorously the statistical mechanics of a gas of vortices in the thermodynamic limit. Thus we let the volume of fluid go to infinity, while the density and mean energy of vortices tend to constants. In this limit, no negative temperature states are found to exist ${ }^{3}$, contrary to Onsager's proposal.

Our main results are presented in Sect. 3 (Theorems 3.1 and 3.2).

\footnotetext{
* Address after Aug. 1982: Theoretical Physics, ETH, CH-8093 Zürich, Switzerland

$\star \star$ Work supported in part by NSF Grant MCS 78-02721

1 The argument of Poincaré [22, Chap. VIII] is based on a discussion of the stability of motion

2 See also Kraichnan and Montgomery [15] for a discussion of this theory. Note that vorticity is conserved in an inviscid fluid (theorem of Helmholtz); therefore Onsager's mechanism cannot explain the appearance of well defined vortices in a fluid where the vorticity is smoothly spread out initially 3 Negative temperature states are known to exist for certain other systems without kinetic energy (spin systems). We claim that nothing of the sort is present here
} 


\section{Potentials}

Let $v=\left(v_{1}, v_{2}\right)$ be the velocity field of an incompressible two-dimensional fluid in a bounded open region $\Lambda \subset \mathbb{R}^{2}$. The incompressibility relation:

$$
\frac{\partial v_{1}}{\partial x_{1}}+\frac{\partial v_{2}}{\partial x_{2}}=0
$$

implies the existence of a stream function $\Psi$ such that

$$
v_{1}=\frac{\partial \Psi}{\partial x_{2}}, v_{2}=-\frac{\partial \Psi}{\partial x_{1}},
$$

if we know that the flow of $v$ through pieces of the boundary of $\Lambda$ vanishes. Introducing the vorticity

$$
\omega=\frac{\partial v_{2}}{\partial x_{1}}-\frac{\partial v_{1}}{\partial x_{2}}=-\Delta \Psi
$$

one sees that the instantaneous angular velocity of a fluid element is $\omega / 2$. The relation (1.1) may be solved for $\Psi$ as

$$
\Psi(x)=\int_{\Lambda} d y \omega(y) V(x, y),
$$

where the potential $V(x, y)$ is the kernel of the operator $V=(-\Delta)^{-1}$, defined with suitable boundary conditions (b.c.) on the space $L^{2}(\Lambda)=L^{2}(\Lambda$, Lebesgue).

We impose the physical condition that the fluid does not cross the boundary of $\Lambda$. If $\partial \Lambda$ is smooth, $v$ is thus parallel to the boundary, and $\operatorname{grad} \Psi$ normal to it. Therefore $\Psi$ is constant on $\partial \Lambda$, and we may take this constant equal to 0 . Mathematically, this corresponds to taking $\Delta=\Delta_{\Lambda}$, where $\Delta_{\Lambda}$ is the Laplacian with Dirichlet b.c. : $-\Delta_{\Lambda}$ is defined as the Friedrichs extension ${ }^{4}$ of the positive operator $-\frac{\partial^{2}}{\partial x_{1}^{2}}-\frac{\partial^{2}}{\partial x_{2}^{2}}$ acting on $C^{\infty}$ functions with compact support in $\Lambda$. The corresponding potential will be denoted by $V_{\Lambda}(x, y)$. We extend the definition of this potential so that $V_{\Lambda}(x, y)=0$ if $x \notin \Lambda$ or $y \notin \Lambda ; V_{\Lambda}(x, y)$ is then the kernel of an operator $V_{\Lambda}$ on $L^{2}\left(\mathbb{R}^{2}\right)$, vanishing on the orthogonal complement of $L^{2}(\Lambda)$. If $\Lambda \subset \Lambda^{\prime}$, the definition of the Friedrichs extension implies that the domain of $\Delta_{A}$ is contained in the domain of $\Delta_{\Lambda^{\prime}}$ [with the identification of $L^{2}(\Lambda)$ to a subspace of $L^{2}\left(\Lambda^{\prime}\right)$ ], and that

$$
\mathbf{1}_{\Lambda}\left(-\Delta_{\Lambda^{\prime}}\right) \mathbf{1}_{\Lambda} \leqq-\Delta_{\Lambda},
$$

where $\mathbf{1}_{\Lambda}$ is the orthogonal projection on $L^{2}(\Lambda)$. Writing

$$
A=\left(-\Delta_{\Lambda^{\prime}}\right)^{1 / 2} V_{\Lambda}^{1 / 2}, A^{*}=V_{\Lambda}^{1 / 2}\left(-\Delta_{A^{\prime}}\right)^{1 / 2} \mathbf{1}_{\Lambda^{\prime}}
$$

we have thus

$$
A^{*} A \leqq \mathbf{1}_{\Lambda}
$$

hence

$$
A A^{*} \leqq \mathbf{1}_{\Lambda^{\prime}}
$$

4 For a discussion of the Friedrichs extension, see for instance Riesz and Nagy [24, Sect. 124] and Reed and Simon [23] 
and finally

$$
V_{\Lambda} \leqq V_{\Lambda^{\prime}} \text { when } \Lambda \subset \Lambda^{\prime} .
$$

It is convenient to introduce also the potential ${ }^{5}$

$$
V_{\infty}(x, y)=-\frac{1}{2 \pi} \log |y-x| .
$$

which corresponds to free b.c. (this is a definition of free b.c.).

If we write

$$
\tilde{V}_{\Lambda}(x, y)=V_{\Lambda}(x, y)-V_{\infty}(x, y),
$$

then $(x, y) \rightarrow \tilde{V}_{\Lambda}(x, y)$ is continuous in $\Lambda \times \Lambda$. [To see this it suffices to notice that $\tilde{V}_{\Lambda}(x, y)$ is a harmonic function of both $x$ and $y$.] We define

$$
W_{\Lambda}(x)=\frac{1}{2} \tilde{V}_{\Lambda}(x, x)
$$

Let $\Lambda$ be fixed, contained in the circle of radius $R$ centered at 0 , and let $\Lambda^{\prime}$ be a circle with large radius $R^{\prime}$ centered at 0 . For $y \in \Lambda, \tilde{V}_{\Lambda^{\prime}}(\cdot, y)$ is harmonic with boundary values $\frac{1}{2 \pi} \log R^{\prime}+O\left(\frac{R}{R^{\prime}}\right)$. Therefore, by the maximum principle

$$
\left|\tilde{V}_{\Lambda^{\prime}}(\cdot, y)-\frac{1}{2 \pi} \log R^{\prime}\right| \leqq O\left(\frac{R}{R^{\prime}}\right) .
$$
When $\omega$ has support in $\Lambda$ and satisfies the "neutrality" condition $\int_{\Lambda} \omega d x=0$, we
have thus

hence

$$
\left|\int \omega(x) \omega(y) \tilde{V}_{A^{\prime}}(x, y) d x d y\right| \leqq O\left(\frac{R}{R^{\prime}}\right)
$$

$$
\lim _{R^{\prime} \rightarrow \infty} \int \omega(x) \omega(y) V_{\Lambda^{\prime}}(x, y) d x d y=\int \omega(x) \omega(y) V_{\infty}(x, y) d x d y .
$$

Combined with (1.2) this gives

$$
\begin{aligned}
\int \omega(x) \omega(y) V_{\Lambda}(x, y) d x d y \leqq & \int \omega(x) \omega(y) V_{\infty}(x, y) d x d y, \\
& \text { when } \int \omega(x) d x=0 .
\end{aligned}
$$

If $\mathscr{L}=\left\{n_{1} a_{1}+n_{2} a_{2}: n_{1}, n_{2} \in \mathbb{Z}\right\}$ is a lattice in $\mathbb{R}^{2}$, and $\Lambda=\left\{\lambda_{1} a_{1}+\lambda_{2} a_{2}\right.$ : $\left.0 \leqq \lambda_{1}, \lambda_{2} \leqq 1\right\}$, a potential $V_{\Lambda \text { per }}(x, y)$ with periodic b.c. may be introduced. It is a periodic function $\varphi$ of $x-y$, with

$$
-\Delta \varphi(\xi)=\sum_{a \in \mathscr{L}} \delta(\xi-a)-|\Lambda|^{-1},
$$

where $|\Lambda|$ is the surface of $\Lambda$. It is seen that $V_{A \text { per }}$ corresponds to the inverse of the Laplacian on a torus, restricted to the orthogonal complement of the constant functions. We have thus

$$
\begin{aligned}
\int \omega(x) \omega(y) V_{\Lambda}(x, y) d x d y \leqq & \int \omega(x) \omega(y) V_{\Lambda \text { per }}(x, y) d x d y, \\
& \text { when } \int \omega(x) d x=0 .
\end{aligned}
$$

5 We write $|x|=\left(x_{1}^{2}+x_{2}^{2}\right)^{1 / 2}$ 
The difference $V_{\Lambda \text { per }}(x, y)-V_{\infty}(x, y)$ is continuous at $x=y$, and we define

$$
W_{\Lambda \mathrm{per}}=\frac{1}{2}\left[V_{\Lambda \mathrm{per}}(x, y)-V_{\infty}(x, y)\right]_{x=y}
$$

which is a constant.
We call $-\Delta_{\Lambda N}$ the operator associated with the quadratic form $\int_{\Lambda} d x \sum_{i}\left(\frac{\partial \varphi}{\partial x_{i}}\right)^{2}$ defined on $C^{\infty}$ functions with bounded derivatives on $\Lambda ; \Delta_{\Lambda N}$ is the Laplacian with Neumann b.c., which corresponds to a vanishing normal derivative on the boundary of $\Lambda$ when this boundary is sufficiently smooth. If $\Lambda \subset \Lambda^{\prime}$ we have $-\Delta_{\Lambda N} \leqq-\Delta_{\Lambda^{\prime} N}$ with the usual identifications. We define $V_{A N}$ to be the inverse of $-\Delta_{\Lambda N}$ restricted to the orthogonal complement of the constant functions on $\Lambda$. The corresponding potential thus satisfies

$$
-\Delta_{\Lambda N} V_{\Lambda N}(\cdot, y)=\delta_{y}-|\Lambda|^{-1} .
$$

Assuming always

$$
\int \omega(x) d x=0,
$$

one obtains easily the following inequalities:

$$
\begin{gathered}
\int \omega(x) \omega(y) V_{\Lambda^{\prime} N}(x, y) d x d y \leqq \int \omega(x) \omega(y) V_{\Lambda N}(x, y) d x d y \text { if } \Lambda \subset \Lambda^{\prime}, \\
\int \omega(x) \omega(y) V_{\infty}(x, y) d x d y \leqq \int \omega(x) \omega(y) V_{\Lambda N}(x, y) d x d y, \\
\int \omega(x) \omega(y) V_{\Lambda \text { per }}(x, y) d x d y \leqq \int \omega(x) \omega(y) V_{\Lambda N}(x, y) d x d y .
\end{gathered}
$$

The difference $V_{A N}(x, y)-V_{\infty}(x, y)$ is continuous at $x=y$, and we define

$$
W_{\Lambda N}(x)=\frac{1}{2}\left[V_{\Lambda N}(x, y)-V_{\infty}(x, y)\right]_{x=y},
$$

which is a continuous function of $x$.

The potentials $V_{A}, V_{\infty}, V_{A \text { per }}, V_{\Lambda N}$ may all be interpreted as two-dimensional electrostatic potentials, $V_{A}$ corresponding to conducting b.c., and $V_{\infty}$ to insulating b.c. on $\partial \Lambda$. If $\Lambda \subset \Lambda^{\prime}$, the electrostatic energy of a distribution of charge in $\Lambda$, with conducting b.c., is less than the energy of the same distribution in $\Lambda^{\prime}$ [inequality (1.2)]. This is because, going from $\Lambda^{\prime}$ to $\Lambda$, one allows the electric field of the given charge distribution to perform work on the freely moving charges of the newly introduced conducting boundary.

\section{Mechanics of Vortices}

The kinetic energy of the fluid contained in $\Lambda$ is

$$
\begin{aligned}
K & =\frac{\varrho}{2} \int_{\Lambda} v^{2}=\frac{\varrho}{2} \int_{A}(\operatorname{grad} \Psi)^{2} \\
& =\frac{\varrho}{2}\left(-\Delta_{\Lambda} \Psi, \Psi\right)=\frac{\varrho}{2}\left(\omega, V_{\Lambda} \omega\right) \\
& =\frac{\varrho}{2} \int_{\Lambda} \omega(x) \omega(y) V_{\Lambda}(x, y) d x d y,
\end{aligned}
$$

where $\varrho$ is the density of the fluid. 
Suppose that $\omega=\sum_{1}^{m} \omega_{i}$, where the $\omega_{i}$ have definite signs, small disjoint supports centered at $\xi_{1}, \ldots, \xi_{m}$, and $\sqrt{\varrho} \int \omega_{i}(x) d x=R_{i}$. Then

$$
\begin{aligned}
K= & \frac{\varrho}{2} \sum_{i=1}^{m} \sum_{j=1}^{m} \int \omega_{i}(x) \omega_{j}(y) V_{\Lambda}(x, y) d x d y \\
\approx & \frac{\varrho}{2} \sum_{i} \int \omega_{i}(x) \omega_{i}(y) V_{\Lambda}(x, y) d x d y \\
& +\sum_{i<j} R_{i} R_{j} V_{\Lambda}\left(\xi_{i}, \xi_{j}\right) .
\end{aligned}
$$

If the supports of the $\omega_{i}$ tend to the points $\xi_{i}$, we have

$$
K-\frac{\varrho}{2} \sum_{i} \int \omega_{i}(x) \omega_{i}(y) V_{\infty}(x, y) d x d y \rightarrow U_{\Lambda}\left(\xi_{1}, \ldots, \xi_{m}\right),
$$

where we have written

$$
U_{\Lambda}\left(\xi_{1}, \ldots, \xi_{m}\right)=\sum_{i} R_{i}^{2} W_{\Lambda}\left(\xi_{i}\right)+\sum_{i<j} R_{i} R_{j} V_{\Lambda}\left(\xi_{i}, \xi_{j}\right)
$$

[remember that $W_{A}$ is given by (1.5)]. We define similarly

$$
U_{\infty}\left(\xi_{1}, \ldots, \xi_{m}\right)=\sum_{i<j} R_{i} R_{j} V_{\infty}\left(\xi_{i}, \xi_{j}\right)
$$

and for a parallelogram $\Lambda$,

$$
\begin{aligned}
& U_{\Lambda \mathrm{per}}\left(\xi, \ldots, \xi_{m}\right) \\
& \quad=\sum_{i} R_{i}^{2} W_{\Lambda \mathrm{per}}+\sum_{i<j} R_{i} R_{j} V_{A \mathrm{per}}\left(\xi_{i}, \xi_{j}\right) .
\end{aligned}
$$

The quantity $U_{\Lambda}\left(\xi_{1}, \ldots, \xi_{m}\right)$ is finite when the $\xi_{i}$ are distinct and inside $\Lambda$. On the other hand $K \rightarrow \infty$ when $m>0$. We view $U_{\Lambda}$ as a renormalized energy of the vortex system; it may take positive or negative values. Note that, as a consequence of (1.2),

$$
U_{\Lambda}\left(\xi_{1}, \ldots, \xi_{m}\right) \leqq U_{\Lambda^{\prime}}\left(\xi_{1}, \ldots, \xi_{m}\right) \text { when } \quad \Lambda \subset \Lambda^{\prime} .
$$

Using (1.6) and (1.7) we obtain also

$$
\begin{aligned}
& U_{\Lambda}\left(\xi_{1}, \ldots, \xi_{m}\right) \leqq U_{\infty}\left(\xi_{1}, \ldots, \xi_{m}\right) \text { when } \sum R_{i}=0, \\
& U_{\Lambda}\left(\xi_{1}, \ldots, \xi_{m}\right) \leqq U_{\Lambda \text { per }}\left(\xi_{1}, \ldots, \xi_{m}\right) \text { when } \sum R_{i}=0 .
\end{aligned}
$$

Since the vortices move with the fluid, by the theorem of Helmholtz, we have

$$
\begin{aligned}
\frac{d}{d t}\left(\begin{array}{l}
\xi_{i 1} \\
\xi_{i 2}
\end{array}\right) & =v\left(\xi_{i}\right)=\left(\begin{array}{c}
\partial_{2} \Psi\left(\xi_{i}\right) \\
-\partial_{1} \Psi\left(\xi_{i}\right)
\end{array}\right)=\left(\begin{array}{rr}
0 & 1 \\
-1 & 0
\end{array}\right) \operatorname{grad} \Psi\left(\xi_{i}\right) \\
& =\left(\begin{array}{rr}
0 & 1 \\
-1 & 0
\end{array}\right) \operatorname{grad} \sum_{j} \int_{\Lambda} d y \omega_{j}(y) V_{\Lambda}\left(\xi_{i}, y\right) \\
& \approx\left(\begin{array}{rr}
0 & 1 \\
-1 & 0
\end{array}\right) \operatorname{grad}_{i}\left[\int_{\Lambda} d y \omega_{i}(y) V_{\Lambda}\left(\xi_{i}, y\right)+\sum_{j \neq i} \frac{R_{j}}{\sqrt{\varrho}} V_{\Lambda}\left(\xi_{i}, \xi_{j}\right)\right] .
\end{aligned}
$$


We admit that if the $i$ th vortex were alone in infinite space, its velocity would vanish (although its internal structure might change with time) ${ }^{6}$. This means that we may replace in the right hand side of $(2.3), V_{A}\left(\xi_{i}, y\right)$ by $V_{A}\left(\xi_{i}, y\right)-V_{\infty}\left(\xi_{i}, y\right)=\tilde{V}_{A}\left(\xi_{i}, y\right)$ [see (1.4)]. Notice that $V_{A}$ is self-adjoint and that its kernel is real (because $-\Delta_{A}$ is a real operator). Therefore $\tilde{V}_{A}(x, y)=\tilde{V}_{A}(y, x)$ and

$$
\left.\operatorname{grad} \tilde{V}_{\Lambda}(x, y)\right|_{x=y=\xi_{i}}=\left.\frac{1}{2} \operatorname{grad} \tilde{V}_{\Lambda}(x, x)\right|_{x=\xi_{i}}=\operatorname{grad} W_{\Lambda}\left(\xi_{i}\right) .
$$

Altogether (2.3) becomes

$$
\sqrt{\varrho} \frac{d}{d t}\left(\begin{array}{l}
\xi_{i 1} \\
\xi_{i 2}
\end{array}\right) \approx\left(\begin{array}{rr}
0 & 1 \\
-1 & 0
\end{array}\right) \operatorname{grad}_{i}\left[R_{i} W_{\Lambda}\left(\xi_{i}\right)+\sum_{j \neq i} R_{j} V_{\Lambda}\left(\xi_{i}, \xi_{j}\right)\right] .
$$

Replacing $\approx$ by $=$, we take this as definition of the motion of point vortices. We have thus

$$
\left.\begin{array}{l}
\sqrt{\varrho} R_{i} \frac{d \xi_{i 1}}{d t}=\frac{\partial U_{A}}{\partial \xi_{i 2}}, \\
\sqrt{\varrho} R_{i} \frac{d \xi_{i 2}}{d t}=-\frac{\partial U_{A}}{\partial \xi_{i 1}} .
\end{array}\right\}
$$

These are Hamiltonian equations ${ }^{7}$ in the $2 m$ variables $\xi_{i \alpha}$ with $i=1, \ldots, m ; \alpha=1,2$. In particular Liouville's theorem implies that the volume element $d \xi=d \xi_{11} \wedge d \xi_{12} \wedge \ldots \wedge d \xi_{m 1} \wedge d \xi_{m 2}$ is preserved under time evolution. The total volume of accessible phase space is in fact finite $\left(=|\Lambda|^{m}\right.$, where $|\Lambda|$ is the surface of $\left.\Lambda\right)$. It is thus natural to follow Onsager, and apply the methods of statistical mechanics to systems of vortices. This means describing systems of many vortices in terms of Gibbs ensembles.

The microcanonical ensemble is the probability measure

$$
\Omega^{-1} \delta\left(U_{\Lambda}\left(\xi_{1}, \ldots, \xi_{m}\right)-E\right) d \xi,
$$

where $\Omega$ is a normalization constant, and the support of the measure is on the energy surface defined by

$$
U_{\Lambda}\left(\xi_{1}, \ldots, \xi_{m}\right)=E
$$

6 A more careful discussion would approximate the velocity field some distance away from the vortex in the form $x \rightarrow v\left(\xi_{i}\right)+\left(\begin{array}{rr}0 & 1 \\ -1 & 0\end{array}\right) \operatorname{grad} V_{\propto}\left(x, \xi_{i}\right)$, and define $v\left(\xi_{i}\right)$ on this basis. Note that an isolated vortex enclosed in a box $\Lambda$ will usually move, due to the presence of walls. In the simple example of a straight infinite wall, we have $V_{A}(x, \xi)=V_{\infty}(x, \xi)-V_{\infty}\left(x, \xi^{s}\right)$, where $\xi^{s}$ is the symmetric of $\xi$ with respect to the wall. The vortex at $\xi$ moves under the influence of its mirror image at $\xi^{s}$. The motion of $\xi, \xi^{s}$ corresponds exactly in 2 dimensions to the motion of a smoke ring in 3 dimensions

7 Writing $q_{i}=\xi_{i 1} \sqrt{\varrho} \sqrt{\left|R_{i}\right|}, p_{i}=\xi_{i 2} R_{i} \sqrt{\varrho} / \sqrt{\left|R_{i}\right|}$, we obtain the familiar equations $d q_{i} / d t=\partial U_{A} / \partial p_{i}$, $d p_{i} / d t=-\partial U_{A} / \partial q_{i}$. It is however more natural to retain the variables $\xi_{i \alpha}$ 
The statistical description of a system by the microcanonical ensemble is usually justified by assuming ergodicity of the measure (2.5) under time evolution ${ }^{8}$.

The heart of statistical mechanics is the study of the thermodynamic limit, where $\Lambda, n, E$ tend to infinity, while the density $|\Lambda|^{-1} n$ and specific energy $|\Lambda|^{-1} E$ have finite limits $\varrho, \varepsilon$. Before taking the limit, one replaces (2.5), where $E$ has a fixed value, by an expression where $E$ varies in an interval $(|\Lambda|(\varepsilon-\delta),|\Lambda|(\varepsilon+\delta))$, and only after the thermodynamic limit does one let $\delta \rightarrow 0$. It is a new physical assumption that this complicated limiting process gives a correct description of (2.5) when $\Lambda, n, E$ are large but finite, and $E$ is a number, not an interval. There is some ambiguity in the limiting regime which is chosen. This will be briefly discussed in Sect. 5.4. In the next sections we go into the formalism of statistical mechanics, and study the thermodynamic limit. Before that let us recapitulate the physical assumptions that have been made:

(a) Two dimensions

(b) No viscosity

(c) Point vortices

(d) Ergodicity of the microcanonical ensemble under time evolution; (or dominance of an ergodic component in the presence of small random perturbations)

(e) Fixed total energy may be replaced by a small energy interval; the thermodynamic limit is a good approximation for the description of large, finite systems at moderate densities and energy.

\section{Statistical Mechanics of Vortices}

3.1. Introductory Remarks. In order to simplify matters, we assume that all vortices have strength $R_{i}= \pm R$, with $R>0$. According to (2.1) the Hamilton function for $n$ positive vortices at positions $\xi_{1}, \ldots, \xi_{n}$ and $m$ negative vortices at positions $\bar{\xi}_{1}, \ldots, \bar{\xi}_{m}$ in an open region $\Lambda \subset \mathbb{R}^{2}$ is given by

$$
\begin{aligned}
U_{A, X}\left(\xi^{n}, \bar{\xi}^{m}\right)= & \sum_{i=1}^{n} R^{2} W_{A, X}\left(\xi_{i}\right)+\sum_{j=1}^{m} R^{2} W_{A, X}\left(\bar{\xi}_{j}\right) \\
& +\sum_{1 \leqq i<l \leqq n} R^{2} V_{A, X}\left(\xi_{i}, \xi_{l}\right)+\sum_{1 \leqq j<k \leqq m} R^{2} V_{A, X}\left(\bar{\xi}_{j}, \bar{\xi}_{k}\right) \\
& -\sum_{\substack{i=1, \ldots, n \\
j=1, \ldots, m}} R^{2} V_{A, X}\left(\xi_{i}, \bar{\xi}_{j}\right),
\end{aligned}
$$

where $X$ specifies the boundary conditions (b.c.), $X=D$ (Dirichlet or conducting b.c.), $X=F$ (free or insulating b.c.), $X=P$ (periodic b.c.), $X=N$ (Neumann b.c.),

8 Nothing is known on the ergodicity of a system of $n$ vortices in a box. (For a discussion of the dynamics of 3 or 4 vortices in infinite space, see Novikov [18], Novikov and Sedov [19], Aref [1], Ziglin [28], and Aref and Pomphrey [2].) Actually, ergodicity may be too strong an assumption. It would be enough to assume that for large $\Lambda, n, E,(2.5)$ has one ergodic component of measure close to 1 . (One may suppose that the other components would be invisible, for instance because points starting in them would, by small random perturbations, go to the large ergodic component) 
$\xi^{n} \equiv\left(\xi_{1}, \ldots, \xi_{n}\right) . \quad$ Furthermore $\quad W_{\Lambda, D} \equiv W_{\Lambda}, \quad V_{\Lambda, D} \equiv V_{\Lambda}, \quad W_{\Lambda, F}=0, \quad V_{\Lambda, F} \equiv V_{\infty}$, $W_{\Lambda, P} \equiv W_{\Lambda \text { per }}$, and $V_{\Lambda, P} \equiv V_{\Lambda \text { per }}$ in the notations of Sects. 1 and 2 .

The system is called neutral if $n=m$. It is straightforward, but cumbersome notationally, to deal with vortices of variable strength, distributed according to some a priori distribution, $d \lambda(R)$, of compact support; (see Appendix B).

It may be of interest to also consider the thermodynamics of "non-neutral" systems, e.g. $n \equiv 0$. Their behaviour differs from the one of neutral systems $(m=n)$ which we study below. In order to obtain thermodynamic behaviour, a neutralizing, uniform background vorticity must be introduced. Physically, such a background vorticity corresponds to a fluid in uniform rotation with constant angular velocity, or one which "shears" between two parallel lines. In this way one obtains a family of vortex systems interpolating between the neutral two-component Coulomb plasma and the "jellium" [26] in which all point vortices have strength $-R$, and there is a neutralizing, uniform positive background vorticity.

For the purpose of comparison (e.g. with numerical studies [17]) we not only discuss the physically motivated Dirichlet-, but also free-, periodic- and Neumann b.c. The remarks on the physics of the vortex system, assumptions (a) through (e), Sect. 2, suggest that we study the micro-canonical ensemble. It turns out, however, that for many values of the thermodynamic parameters this ensemble is equivalent to the canonical ensemble. Moreover, for the system of point vortices studied below, the canonical ensemble is known to be equivalent to the grand canonical ensemble. This is a simple consequence of the scaling properties of $U_{A, X}$ (see Sect. 4 of [8], [9], and Theorem 3.2). Mathematically, the grand canonical ensemble is the most convenient one.

\subsection{Definition of Ensembles and Thermodynamic Functions, The Main Results}

(a) Microcanonical Ensembles. Let $\delta^{\Delta}$ be the characteristic function of the interval $[-\Delta, 0]$ and $\delta^{-}$the one of $(-\infty, 0]$. The microcanonical partition function for a neutral system of $n$ positive and $n$ negative vortices in a bounded, open domain $\Lambda \subset \mathbb{R}^{2}$ is given by

$$
\Omega^{\Delta, X}(\Lambda, n, E)=\left(\frac{1}{n !}\right)^{2} \int_{\Lambda^{2 n}} \delta^{\Delta}\left(U_{\Lambda, X}\left(\xi^{n}, \bar{\xi}^{n}\right)-E\right) d \xi^{n} d \bar{\xi}^{n},
$$

where $E$ is the total energy, and

$$
d \xi^{n}=\prod_{i=1}^{n} d^{2} \xi_{i}, d \bar{\xi}^{n}=\prod_{j=1}^{n} d^{2} \bar{\xi}_{j}
$$

(We closely follow notation in $[8,9,25]$.) We also define

$$
\Omega^{X}(\Lambda, n, E)=\left(\frac{1}{n !}\right)^{2} \int_{\Lambda^{2 n}} \delta^{-}\left(U_{\Lambda, X}\left(\xi^{n}, \bar{\xi}^{n}\right)-E\right) d \xi^{n} d \bar{\xi}^{n}
$$


Let

$$
\varepsilon \equiv \frac{E}{|\Lambda|}, \delta \equiv \frac{\Delta}{|\Lambda|}, \varrho \equiv \frac{2 n}{|\Lambda|},
$$

where $|\Lambda|$ is the "volume" (area) of $\Lambda$. These quantities are the energy density, energy density uncertainty and particle density, respectively. We define the entropy densities

$$
\begin{aligned}
s_{\Lambda}^{\delta, X}(\varrho, \varepsilon) & \equiv \frac{1}{|\Lambda|} \log \Omega^{\Lambda, X}(\Lambda, n, E), \\
s_{\Lambda}^{X}(\varrho, \varepsilon) & \equiv \frac{1}{|\Lambda|} \log \Omega^{X}(\Lambda, n, E)>s_{\Lambda}^{\delta, X}(\varrho, \varepsilon),
\end{aligned}
$$

and

$$
s^{\delta, X}(\varrho, \varepsilon)=\lim _{\Lambda>\mathbb{R}^{2}} s_{\Lambda}^{\delta, X}(\varrho, \varepsilon), s^{X}(\varrho, \varepsilon) \equiv \lim _{\Lambda \gg \mathbb{R}^{2}} s_{\Lambda}^{X}(\varrho, \varepsilon),
$$

with $\delta, \varepsilon$, and $\varrho$ kept fixed, ( $\Lambda \nearrow \mathbb{R}^{2}$ in the sense of van Hove [25]). For Neumann b.c. the thermodynamic limit of $s_{\Lambda}^{N}$ can be shown to exist (see Sect. 4). The function $s_{\Lambda}^{X}(\varrho, \varepsilon)$ is by definition an increasing function of $\varepsilon$.

It is easy to see [25] that if the thermodynamic limit of $s_{\Lambda}^{X}(\varrho, \varepsilon)$ exists and if $s^{X}(\varrho, \varepsilon)$ is strictly increasing at some $\varepsilon=\varepsilon_{0}$, then

$$
\lim _{\Lambda \supset \mathbb{R}^{2}} s_{\Lambda}^{\delta, X}\left(\varrho, \varepsilon_{0}\right)=s^{\delta, X}\left(\varrho, \varepsilon_{0}\right)=s^{X}\left(\varrho, \varepsilon_{0}\right),
$$

for all $\delta>0$. It might happen, however, that $s^{X}(\varrho, \varepsilon)=s_{0}=$ const, for $\varepsilon \in\left[\varepsilon_{0}, \varepsilon_{1}\right]$, $\varepsilon_{0}<\varepsilon_{1}$. In that case it is conceivable that $s^{\delta, X}(\varrho, \varepsilon)$ depends on $\delta$ and is strictly smaller than $s(\varrho, \varepsilon)$, for some $\varepsilon \in\left(\varepsilon_{0}, \varepsilon_{1}\right)$ and some sufficiently small $\delta$. Thus it might happen that

$$
\frac{\partial s^{\delta, X}(\varrho, \varepsilon)}{\partial \varepsilon} \equiv \beta<0
$$

at energy densities $\varepsilon$ around which $s^{X}(\varrho, \cdot)$ is constant. This was, in fact, expected by Onsager [20]. Of course, in a finite region $\Lambda$,

$$
\frac{\partial s_{\Lambda}^{\delta, X}(\varrho, \varepsilon)}{\partial \varepsilon}<0
$$

if $\varepsilon$ is large enough, depending on $\Lambda$. The true behaviour of $s^{\delta, X}(\varrho, \varepsilon), X=D, F, P$, or $N$, as a function of $\varepsilon$ is described in the following result.

Theorem 3.1. For $X=D, F, P, N$, there exists a function $\sigma^{X}(\varrho, \varepsilon)$ such that

$$
\varepsilon \rightarrow \sigma^{X}(\varrho, \varepsilon)
$$
is increasing and concave in $\varepsilon$, with values in the open interval $\left(-\infty, \varrho-\varrho \log \frac{\varrho}{2}\right)$, and
if $\sigma^{X}(\varrho, \cdot)$ is strictly concave at $\varepsilon$

$$
s^{\delta, X}(\varrho, \varepsilon)=s^{X}(\varrho, \varepsilon)=\sigma^{X}(\varrho, \varepsilon) .
$$


(In particular, the thermodynamic limits of $s_{\Lambda}^{\delta, X}$ and $s_{\Lambda}^{X}$ exist.) If $\varepsilon$ belongs to an interval of linearity $\left(\varepsilon_{R}, \varepsilon_{L}\right)$ of $\sigma^{X}(\varrho, \cdot)$ then

$$
\begin{gathered}
\varlimsup_{\Lambda \rtimes \mathbb{R}^{2}} s_{\Lambda}^{X}(\varrho, \varepsilon) \leqq \sigma^{X}(\varrho, \varepsilon), \\
\varlimsup_{\Lambda \rtimes \mathbb{R}^{2}} s^{\delta, X}(\varrho, \varepsilon) \geqq \sigma^{X}\left(\varrho, \varepsilon_{R}\right) .
\end{gathered}
$$

(Note that $\varepsilon_{R}$ is the left extremity of the interval.) Finally

$$
\lim _{\varepsilon \rightarrow-\infty} \sigma^{X}(\varrho, \varepsilon)=-\infty, \lim _{\varepsilon \rightarrow+\infty} s^{\delta, X}(\varrho, \varepsilon)=\varrho-\varrho \log \frac{\varrho}{2} .
$$

Notice that

$$
\varrho-\varrho \log \frac{\varrho}{2}=\lim _{\Lambda>\mathbb{R}^{2}} \frac{1}{|\Lambda|} \log \left[\left(\frac{1}{n !}\right)^{2}|\Lambda|^{2 n}\right], \varrho=\frac{2 n}{|\Lambda|},
$$

is the entropy of an ideal (non-interacting), two-component gas of vortices. A proof of (3.5) and (3.6) is given in Appendix A. The remaining statements then follow by proving the equivalence of the microcanonical, canonical, and grand canonical ensemble for all, but possibly countably many values of

$$
\beta=\frac{\partial \sigma^{X}(\varrho, \varepsilon)}{\partial \varepsilon},
$$

and exploiting detailed properties of the free energy as a function of $\varrho$ and $\beta$; see Theorem 3.2 and Sects. 4 and 5.

In Sect. 4 we show

$$
s^{N}(\varrho, \varepsilon) \leqq s^{X}(\varrho, \varepsilon) \leqq s^{D}(\varrho, \varepsilon)<\varrho-\varrho \log \frac{\varrho}{2},
$$

for all $\varepsilon<\infty$ and $X=F$ or $P$.

Using a conjectured extension of the results in $[4,5]$ (proven for a lattice Coulomb gas) to the continuum gas studied here, one is able to establish the equivalence of all three ensembles, for $\beta$ sufficiently small, i.e. $\varepsilon$ sufficiently large, and Dirichlet boundary conditions, and to exhibit the approach of $s^{D}(\varrho, \varepsilon)$ to $\varrho-\varrho \log \frac{\varrho}{2}$, as $\varepsilon \rightarrow \infty$, explicitly (see Sect. 5).

In the next section we establish some general properties of $s^{\delta}(\varrho, \varepsilon)$ and $s(\varrho, \varepsilon)$; in particular, we prove the following scaling relation: For arbitrary b.c. and all $\theta>0$,

$$
s_{\Lambda}\left(\varrho^{\prime}, \varepsilon^{\prime}\right)=2 \varrho^{\prime} \log \theta+\theta^{-2} s_{\theta^{-1} \Lambda}\left(\theta^{2} \varrho^{\prime}, \theta^{2} \varepsilon^{\prime}+\frac{\varrho^{\prime} R^{2}}{4 \pi} \theta^{2} \log \theta\right),
$$

which can be transferred to the thermodynamic limit if the latter exists. In that case we obtain

$$
S(\varrho, \varepsilon)=e^{8 \pi \varepsilon / \varrho R^{2}} S\left(e^{-8 \pi \varepsilon / \varrho R^{2}} \varrho, 0\right)-8 \pi \varepsilon / \varrho R^{2},
$$

by choosing $\varepsilon^{\prime}=0, \varrho^{\prime}=\theta^{-2} \varrho, \theta=e^{4 \pi \varepsilon / \varrho R^{2}}$.

Thus, the entropy as a function of $\varrho$ and $\varepsilon$ is determined by the entropy as a function of $\varrho$, for a fixed value, $\varepsilon_{0}$, for example 0 , of the energy density $\varepsilon$, provided 
the thermodynamic limit exists (see Sect. 4 and Appendix A.) Existence of the thermodynamic limit can be proven for strictly neutral systems with Neumann b.c. (Sect. 4). This summarizes our main results, but see also Theorem 3.2 and (3.34).

(b) Canonical and Grand Canonical Ensembles. The canonical partition function for a system of $n$ positive and $m$ negative vortices in a bounded, open domain $\Lambda \subset \mathbb{R}^{2}$, with $D, F, P$ or $N$ boundary conditions at $\partial \Lambda$, is defined by

$$
Q^{X}(\Lambda, n, m, \beta)=\frac{1}{n !} \cdot \frac{1}{m !} \int_{\Lambda^{n+m}} \cdot d \xi^{n} d \bar{\xi}^{m} e^{-\beta U_{\Lambda, X}\left(\xi^{n}, \bar{\xi}^{m}\right)},
$$

with $U_{A, X}$ given by (3.1), and

$$
Q^{X}(\Lambda, n, m, \beta) \equiv 0, \text { unless } n=m,
$$

if $X=N, F$ or $P$ b.c. are imposed. We define

$$
Q^{X}(\Lambda, n, \beta) \equiv Q^{X}(\Lambda, n, n, \beta),
$$

and

$$
\beta f_{\Lambda}^{X}(\varrho, \beta) \equiv-\frac{1}{|\Lambda|} \log Q^{X}(\Lambda, n, \beta)
$$

with $\varrho=\frac{2 n}{|\Lambda|} ; f_{\Lambda}^{X}(\varrho, \beta)$ is the free energy density for a neutral system in $\Lambda$ with b.c. $X$.

It is proven in $[8,9]$, Sects. 3 and 4 , that for $0<\beta<\frac{4 \pi}{R^{2}}$ and $\frac{n}{|\Lambda|}$ bounded,

$$
Q^{X}(\Lambda, n, m, \beta) \leqq K(\beta)^{n+m},
$$

and

$$
Q^{X}(\Lambda, n, n, \beta)=\infty, \quad \text { for } \quad \beta \geqq \frac{4 \pi}{R^{2}}
$$

for $X=F, P$, and $N$. In Appendix B, this result is extended to $X=D$ (in which case (3.12) has been shown to hold for $\beta<\frac{2 \pi}{R^{2}}$ in [9]).

By using an argument of Griffiths [12], it has been observed in [13] that

$$
f^{F}(\varrho, \beta)=\lim _{\Lambda \rtimes \mathbb{R}^{2}} f_{\Lambda}^{F}(\varrho, \beta)
$$

(where the limit is understood in the sense of van Hove; see Definition 2.1.1 in [25]). The same argument works for Neumann b.c. [the important ingredient in the proof being inequality (1.8)].

For all four choices of b.c. the existence of the thermodynamic limit of $f_{\Lambda}^{X}(\varrho, \beta)$ can also be deduced from the equivalence of the canonical and the grand canonical ensemble and the existence of the thermodynamic limit of the pressure for all $0<\beta<\frac{4 \pi}{R^{2}}$ and $X=D, F, P, N$; (see Sect. 5). 
We now define the grand partition function

$$
\begin{gathered}
\Xi^{X}(\Lambda, z, \beta)=\sum_{n, m=0}^{\infty} z^{n+m} Q^{X}(\Lambda, n, m, \beta), \\
Q^{X}(\Lambda, 0,0, \beta) \equiv 1,
\end{gathered}
$$

where $z \equiv e^{\beta \mu}$ is the activity, $0 \leqq z<\infty$. The pressure is then given by

$$
\beta p_{\Lambda}^{X}(z, \beta)=\frac{1}{|\Lambda|} \log \Xi^{X}(\Lambda, z, \beta) .
$$

In Sect. 5 we show that for $\beta<\frac{4 \pi}{R^{2}}$

$$
0<p_{\Lambda}^{N}(z, \beta) \leqq\left(\begin{array}{l}
p_{\Lambda}^{F}(z, \beta) \\
p_{\Lambda}^{P}(z, \beta)
\end{array}\right) \leqq p_{\Lambda}^{D}(z, \beta) \leqq K(z, \beta),
$$

where $K(z, \beta)$ is some finite constant independent of $\Lambda$, provided $\Lambda$ is a circular or rectangular region containing $\{\xi:|\xi| \leqq 1\}$. Moreover, for $X=F$ and $N$ and $\{\Lambda\}$ an increasing sequence of circular or rectangular regions

$$
\left.\begin{array}{l}
p^{X}(z, \beta) \text { is monotone increasing in } \Lambda, \\
p^{D}(z, \beta) \text { is monotone decreasing in } \Lambda .
\end{array}\right\}
$$

By (3.16) and (3.17),

$$
p^{X}(z, \beta)=\lim _{\Lambda>\mathbb{R}^{2}} p_{\Lambda}^{X}(z, \beta)
$$

exists and is finite and positive, for all $0<z<\infty, 0<\beta<\frac{4 \pi}{R^{2}}$, and $X=D, F, N$.

One can also establish (3.18) for $X=P$, with $\Lambda \nearrow \mathbb{R}^{2}$ through a sequence of squares or rectangles (see Sect. 5 and [6]).

By (3.16) and (3.17), for $X=F, N$,

$$
0<p_{\Lambda}^{X}(z, \beta)<p^{X}(z, \beta) \leqq p^{D}(z, \beta)<p_{\Lambda}^{D}(z, \beta) .
$$

By (3.10), (3.14), and (3.15)

$$
\lim _{\beta>0} \beta p_{\Lambda}^{X}(z, \beta)=p_{\Lambda}^{0}(z) \equiv \frac{1}{|\Lambda|} \log \left(\sum_{n=0}^{\infty} \frac{(z|\Lambda|)^{2 n}}{(n !)^{2}}\right),
$$

for $X=F, P$, and $N$, and

$$
\lim _{\beta \rightarrow 0} \beta p_{\Lambda}^{D}(z, \beta)=p_{\Lambda}^{1}(z) \equiv \frac{1}{|\Lambda|} \log \left(\sum_{n, m=0}^{\infty} \frac{(z|\Lambda|)^{n+m}}{n ! m !}\right)=2 z .
$$

It is an elementary fact that

$$
\lim _{\Lambda \rtimes \mathbb{R}^{2}} p_{\Lambda}^{0}(z)=2 z
$$

By (3.19) and (3.20)

$$
p_{\Lambda}^{0}(z)<\lim _{\beta \rightarrow 0} \beta p^{X}(z, \beta) \leqq 2 z
$$


for $X=D, F, P, N$ and arbitrary $\Lambda$, so that by (3.21),

$$
\left.\begin{array}{l}
\lim _{\beta>0} \beta p^{X}(z, \beta)=2 z=\lim _{\beta>0} \beta p_{\Lambda}^{D}(z, \beta), \\
\text { for } X=D, F, P, N \text { and for all } \quad \Lambda .
\end{array}\right\}
$$

Next, by (3.10), (3.14), and (3.15),

$$
\begin{aligned}
\frac{\partial\left(\beta p_{\Lambda}^{D}(z, \beta)\right)}{\partial \beta}= & -\frac{R^{2}}{|\Lambda|} \int_{\Lambda} d^{2} \xi W_{\Lambda}(\xi) \varrho_{\Lambda}(\beta, z ; \xi) \\
& -\frac{R^{2}}{|\Lambda|} \int_{\Lambda \times \Lambda} d^{2} \xi d^{2} \eta V_{\Lambda}(\xi, \eta)\left[\varrho_{\Lambda}^{++}(\beta, z ; \xi, \eta)-\varrho_{\Lambda}^{+-}(\beta, z ; \xi, \eta)\right]
\end{aligned}
$$

where $\varrho_{A}(\beta, z ; \xi)$ is the one-vortex correlation, and $\varrho_{A}^{+ \pm}(\beta, z ; \xi, \eta)$ is the correlation of a vortex with strength $R$ at $\xi$ and a vortex with strength $\pm R$ at $\eta$, in the grand canonical ensemble; see $[25,10]$ for definitions. In a bounded region $\Lambda$,

$$
\lim _{\beta>0}\left[\varrho_{A}^{++}(\beta, z ; \xi, \eta)-\varrho_{A}^{+-}(\beta, z ; \xi, \eta)\right]=0,
$$

and

$$
\lim _{\beta>0} \varrho_{\Lambda}(\beta, z ; \xi)=z .
$$

Thus, by a dominated convergence argument,

$$
\begin{aligned}
\lim _{\beta>0} \frac{\partial\left(\beta p_{\Lambda}^{D}(z, \beta)\right)}{\partial \beta} & =-\frac{z R^{2}}{|\Lambda|} \int_{\Lambda} d^{2} \xi W_{\Lambda}(\xi) \\
& =\frac{z R^{2}}{2|\Lambda|} \int_{\Lambda} d^{2} \xi \lim _{\eta \rightarrow \xi}\left(V_{\infty}(\xi-\eta)-V_{\Lambda}(\xi, \eta)\right) .
\end{aligned}
$$

Suppose now that $\Lambda$ is the disc of radius $r$ centered at the origin. Then

$$
\lim _{\eta \rightarrow \xi}\left(V_{\infty}(\xi-\eta)-V_{\Lambda}(\xi, \eta)\right)=(1 / 4 \pi) \log r^{2}-(1 / 2 \pi) \log \left(r^{2}-\xi^{2}\right) ;
$$

see Sect. 3 of [8], or [9]. Therefore

Hence

$$
\begin{aligned}
\lim _{\beta>0} \frac{\partial\left(\beta p_{\Lambda}^{D}(z, \beta)\right)}{\partial \beta} & =\frac{1}{2} z R^{2}\left[(1 / 4 \pi) \log r^{2}+\frac{1}{2 \pi r^{2}} \int_{0}^{r}(-2 t) \log \left(r^{2}-t^{2}\right) d t\right] \\
& =-\frac{z R^{2}}{8 \pi}\left[\log r^{2}-2\right] .
\end{aligned}
$$

$$
\lim _{\beta>0} \frac{\partial\left(\beta p_{\Lambda}^{D}(z, \beta)\right)}{\partial \beta} \text { diverges to }-\infty \text {, as } r \rightarrow \infty .
$$

By (3.17) and (3.22),

$$
\lim _{\beta>0} \frac{\partial\left(\beta p^{D}(z, \beta)\right)}{\partial \beta}<\lim _{\beta>0} \frac{\partial\left(\beta p_{\Lambda}^{D}(z, \beta)\right)}{\partial \beta},
$$


for arbitrary $\Lambda$. Therefore we conclude that

$$
\lim _{\beta>0} \frac{\partial\left(\beta p^{D}(z, \beta)\right)}{\partial \beta}=-\infty .
$$

Theorem 3.2. Let $\alpha=\beta R^{2} / 4 \pi$. Then, for all choices of b.c., $X=D, F, P, N$,

$$
\beta p^{X}(z, \beta)=z^{2 / 2-\alpha} F^{X}(\alpha),
$$

where $F^{X}(\alpha)$ is a finite, strictly positive convex function of $\alpha$, for $0 \leqq \alpha<1$, with the following properties:

1) $\lim _{\alpha \rightarrow 1} F^{X}(\alpha)=\infty$,

2) $\lim _{\alpha>0} F^{X}(\alpha)=2$,

3) $\lim _{\alpha>0} \frac{d}{d \alpha} F^{X}(\alpha)=-\infty$.

Moreover, the canonical and grand canonical ensembles are equivalent, for all $\beta \in\left[0, \frac{4 \pi}{R^{2}}\right)$, and

$$
\beta f^{X}(\beta, \varrho)=\frac{2-\alpha}{2}\left[\varrho \log \left\{\frac{(2-\alpha) \varrho}{2 F^{X}(\alpha)}\right\}-1\right] .
$$

Remarks. 1) For free b.c., (3.27) and (3.28) are proven in [8]. The extension to other b.c. is indicated in Sect. 5. From (3.27) one derives the equation of state

$$
p^{X}(\varrho, \beta)=(\varrho / \beta)\left(1-\beta R^{2} / 8 \pi\right) ;
$$

see $[16,8,9]$.

2) Assuming that the methods of $[4,5]$ extend to the continuum Coulomb gas, for sufficiently small, but positive values of $\alpha$, one can show that $F^{D}(\alpha)$ is $C^{\infty}$ in $\alpha$, for small $\alpha$, and

$$
\frac{d}{d \alpha} F^{D}(\alpha)=O(\log \alpha)
$$

as $\alpha \gg 0$; see Sect. 5 .

We now turn to the proof of Theorem 3.2. For the proof of Eq. $(3.27)$ see $[8,9]$ and Sect. 5. It follows from (3.10), (3.14), and (3.15) by using Hölder's inequality that $\beta p^{X}(z, \beta)$ is convex in $\beta$, hence in $\alpha$. Thus, using (3.27)

$$
\begin{aligned}
0 \leqq & \frac{\partial^{2}\left(\beta p^{X}(z, \beta)\right)}{\partial \alpha^{2}}=\frac{4}{(2-\alpha)^{3}}(\log z) \beta p^{X}(z, \beta) \\
& +\frac{2}{(2-\alpha)^{2}} \log z\left[\frac{2}{(2-\alpha)^{2}}(\log z) \beta p^{X}(z, \beta)+2 z^{2 / 2-\alpha} F^{\prime}(\alpha)\right] \\
& +z^{2 / 2-\alpha} F^{\prime \prime}(\alpha)=F^{\prime \prime}(\alpha), \quad \text { for } \quad z=1 .
\end{aligned}
$$

Thus $F(\alpha) \equiv F^{X}(\alpha)$ is convex. That it is finite for $0 \leqq \alpha<1$ follows directly from (3.16), and that it diverges when $\alpha \succ 1$ follows from (3.10) and (3.13). [All quantities, 
$Q^{X}, \Xi^{X}$, and $p^{X}$, diverge when $\beta>\frac{4 \pi}{R^{2}}$, because of the logarithmic singularity of $V_{\Lambda}^{X}(\xi, \eta)$ at $\xi=\eta \notin \partial \Lambda$ (see $\left.[8,9]\right)$.]

By (3.22) and (3.27),

$$
\lim _{\beta>0} \beta p^{X}(z, \beta)=z F^{X}(0)=2 z \text {, i.e. } F^{X}(0)=2, \text { for all } X .
$$

This proves property 2) of $F^{X}$. Next,

$$
\begin{aligned}
\frac{\partial}{\partial \beta}\left(\beta p^{D}(z, \beta)\right) & =\frac{R^{2}}{4 \pi} \frac{\partial}{\partial \alpha}\left(\beta p^{D}(z, \beta)\right) \\
& =\frac{R^{2}}{4 \pi}\left[\frac{2}{(2-\alpha)^{2}}(\log z) \beta p^{D}(z, \beta)+z^{2 / 2-\alpha} \frac{d F^{D}(\alpha)}{d \alpha}\right] .
\end{aligned}
$$

This identity combined with (3.26) yield property 3 ), for $X=D$. In order to prove 3 ) for $X=F, P$, and $N$, suppose first that

$$
\frac{d F^{X}(\alpha)}{d \alpha} \geqq \text { const }>-\infty
$$

uniformly in $\alpha \in(0,1)$. Then $\frac{\partial}{\partial \beta}\left(\beta f^{X}(\varrho, \beta)\right) \leqq \varepsilon_{0}<\infty$, for some constant $\varepsilon_{0}$ and all $\beta \in\left(0, \frac{4 \pi}{R^{2}}\right)$. Since by $(3.28)$

$$
\lim _{\beta>0}\left(\beta f^{X}(\varrho, \beta)\right)=\varrho\left(\log \frac{\varrho}{2}-1\right),
$$

and since the entropy density $s^{X}(\varrho, \varepsilon)$ is increasing in $\varepsilon$, we conclude that $s^{X}(\varrho, \varepsilon)=\varrho\left(1-\log \frac{\varrho}{2}\right)$, for all $\varepsilon \geqq \varepsilon_{0}, X=F, P, N$. But, by Theorem 3.1,

$$
s^{D}(\varrho, \varepsilon) \leqq \sigma^{D}(\varrho, \varepsilon)<\varrho\left(1-\log \frac{\varrho}{2}\right),
$$

for all $\varepsilon<\infty$. Actually, the upper bound on $\sigma^{D}$ follows from properties 2) and 3) of $F^{D}$ and Eq. (3.27), Theorem 3.2, as shown below. Thus $s^{X}(\varrho, \varepsilon)>s^{D}(\varrho, \varepsilon)$, for $\varepsilon \in\left(\varepsilon_{0}, \infty\right), X=F, P$ or $N$. This however contradicts inequality (3.7). We therefore conclude that

$$
\lim _{\alpha>0} \frac{d F^{X}(\alpha)}{d \alpha}=-\infty
$$

for all $X$. This completes the proof of property 3). Finally, we observe that by (3.27), $p^{X}(z, \beta)$ is analytic in $z$, except at $z=0, \infty$, and strictly convex on $(0, \infty)$. As is well known (see [25]), this entails the equivalence of the grand canonical and the canonical ensemble. Equation (3.28) therefore follows from (3.27) by Legendre transformation (see Sect. 4 of [8], or [9]). 
The contents of Theorem 3.2 may be summarized, in terms of the free energy density $f^{X}(\varrho, \beta)$, by means of the following Fig. 1 :

Fig. 1

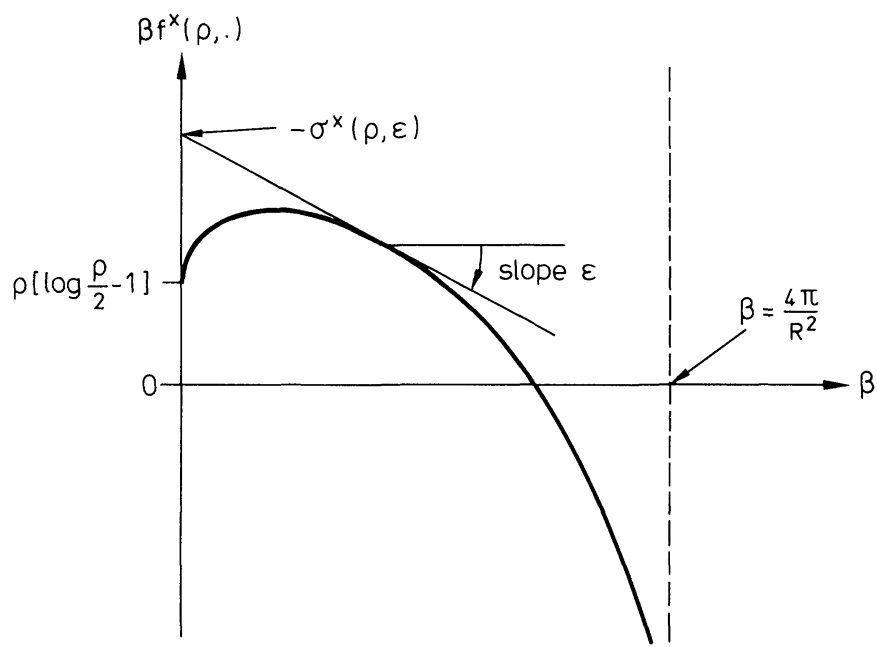

Proof of Theorem 3.1. The main part of Theorem 3.1 is proven in Appendix A. The function $\sigma^{X}(\varrho, \varepsilon)$ is defined by

$$
\sigma^{X}(\varrho, \varepsilon)=\inf _{\beta \in\left(0, \frac{4 \pi}{R^{2}}\right)}\left(\beta \varepsilon-\beta f^{X}(\varrho, \beta)\right) .
$$

Suppose $\beta f^{X}(\varrho, \beta)$ is continuously differentiable at some value $\beta_{0}$ of $\beta$. Then

$$
\sigma^{X}\left(\varrho, \varepsilon_{0}\right)=\left[\beta \frac{\partial\left(\beta f^{X}\right)}{\partial \beta}(\varrho, \beta)-\beta f^{X}(\varrho, \beta)\right]_{\beta=\beta_{0}},
$$

and $\varepsilon_{0}$ is determined by the equation

$$
\varepsilon_{0}=\left[\frac{\partial\left(\beta f^{X}\right)}{\partial \beta}(\varrho, \beta)\right]_{\beta=\beta_{0}} .
$$

Moreover, the microcanonical and the canonical ensemble are equivalent at those values of $\beta$ and $\varepsilon$, and

$$
\lim _{\Lambda>\mathbb{R}^{2}} s_{\Lambda}^{\delta, X}\left(\varrho, \varepsilon_{0}\right)=\lim _{\Lambda>\mathbb{R}^{2}} s_{\Lambda}^{X}\left(\varrho, \varepsilon_{0}\right)=\sigma^{X}\left(\varrho, \varepsilon_{0}\right) .
$$

By properties 2) and 3) of $F^{D}$ and Eq. (3.28) - which we have established without using Theorem 3.1 - there exists a sequence $\left\{\beta_{n}\right\}$ converging to 0 such that $\beta f^{D}(\varrho, \beta)$ is strictly concave and continuously differentiable at $\beta=\beta_{n}$, for all $n$. [By (3.28), $\left\{\beta_{n}\right\}$ can be chosen to be independent of $\varrho$ !] By property 3 ) of $F^{D}$, Theorem 3.2 ,

$$
\varepsilon_{n}=\left[\frac{\partial\left(\beta f^{D}\right)}{\partial \beta}(\varrho, \beta)\right]_{\beta=\beta_{n}} \rightarrow \infty,
$$


as $n \rightarrow \infty$. Therefore $\left\{\sigma^{D}\left(\varrho, \varepsilon_{n}\right)\right\}$ is a strictly increasing sequence, and by property 2) of $F^{D}$, Theorem 3.2,

$$
\lim _{n \rightarrow \infty} \sigma^{D}\left(\varrho, \varepsilon_{n}\right)=\sup _{n} \sigma^{D}\left(\varrho, \varepsilon_{n}\right)=\varrho\left[1-\log \frac{\varrho}{2}\right] .
$$

Using inequality (3.5), Theorem 3.1, which is established in Appendix A, we thus obtain

$$
\varlimsup_{\Lambda \rightarrow \mathbb{R}^{2}} s_{\Lambda}^{D}(\varrho, \varepsilon) \leqq \sigma^{D}(\varrho, \varepsilon)<\varrho\left[1-\log \frac{\varrho}{2}\right],
$$

for all $\varepsilon<\infty$, and using (3.6)

$$
\begin{aligned}
\lim _{n \rightarrow \infty} \lim _{\Lambda>\mathbb{R}^{2}} s_{\Lambda}^{\delta, D}\left(\varrho, \varepsilon_{n}\right) & =\lim _{n \rightarrow \infty} \lim _{\Lambda>\mathbb{R}^{2}} s_{\Lambda}^{D}\left(\varrho, \varepsilon_{n}\right) \\
& =\lim _{n \rightarrow \infty} \sigma^{D}\left(\varrho, \varepsilon_{n}\right)=\varrho\left[1-\log \frac{\varrho}{2}\right] .
\end{aligned}
$$

Next, by property 1) of $F^{D}$, Theorem 3.2 , there exists a sequence $\left\{\beta_{n}^{\prime}\right\}$ converging to $\frac{4 \pi}{R^{2}}$ such that

$$
\lim _{n \rightarrow \infty} \beta_{n}^{\prime} f^{D}\left(\beta_{n}^{\prime}, \varrho\right)=-\infty,
$$

and $\beta f^{D}(\beta, \varrho)$ is strictly concave and continuously differentiable at $\beta=\beta_{n}^{\prime}$. (Again $\left\{\beta_{n}^{\prime}\right\}$ may be chosen to be independent of $\varrho$.) Thus

$$
\varepsilon_{n}^{\prime} \equiv\left[\frac{\partial\left(\beta f^{D}\right)}{\partial \beta}(\beta, o)\right]_{\beta=\beta_{n}^{\prime}}
$$

tends to $-\infty$, as $n \rightarrow \infty$. In conclusion

$$
\begin{aligned}
\lim _{n \rightarrow \infty} \sigma^{D}\left(\varrho, \varepsilon_{n}^{\prime}\right) & =\lim _{n \rightarrow \infty} \lim _{\Lambda \rightarrow \mathbb{R}^{2}} s_{\Lambda}^{D}\left(\varrho, \varepsilon_{n}^{\prime}\right) \\
& =\lim _{n \rightarrow \infty} \lim _{\Lambda \rightarrow \mathbb{R}^{2}} s_{\Lambda}^{\delta, D}\left(\varrho, \varepsilon_{n}^{\prime}\right)=-\infty .
\end{aligned}
$$

This, together with (3.5) and (3.6) (proven in Appendix A), completes the proof of Theorem 3.1 for $X=D$. By inequality (3.7) (which is proven in the next section)

$$
S^{X}(\varrho, \varepsilon) \leqq S^{D}(\varrho, \varepsilon),
$$

for all $\varepsilon<\infty, X=F, P, N$. Thus $\sigma^{X}, s^{X}$, and $s^{\delta, X}$ tend to $-\infty$, as $\varepsilon \rightarrow-\infty$, and

for all $\varepsilon<\infty$.

$$
s^{\delta, X}(\varrho, \varepsilon) \leqq S^{X}(\varrho, \varepsilon)<\varrho\left[1-\log \frac{\varrho}{2}\right],
$$

As already noted in the proof of Theorem 3.2, inequalities (3.22) and (3.32) yield properties 1)-3) of $F^{X}, X=F, P, N$, stated in Theorem 3.2. By repeating the arguments given above for $X=D$, we thus conclude that, for each choice of $X=F$, $P$ or $N$, there exists a sequence $\left\{\varepsilon_{n}\right\}$ diverging to $+\infty$ such that

$$
\begin{aligned}
\lim _{n \rightarrow \infty} \lim _{\Lambda>\mathbb{R}^{2}} s_{\Lambda}^{\delta, X}\left(\varrho, \varepsilon_{n}\right) & =\lim _{n \rightarrow \infty} \lim _{\Lambda \rightarrow \mathbb{R}^{2}} s_{\Lambda}^{X}\left(\varrho, \varepsilon_{n}\right) \\
& =\lim _{n \rightarrow \infty} \sigma_{\Lambda}^{X}\left(\varrho, \varepsilon_{n}\right)=\varrho\left[1-\log \frac{\varrho}{2}\right] .
\end{aligned}
$$

This completes the proof of Theorem 3.1. 
All as yet unproven statements about entropy, free energy or pressure are established in Sects. 4 and 5 and Appendices A and B.

Next, we recall some facts and properties of correlation functions of the twocomponent Coulomb gas in the grand canonical ensemble. (We believe that the properties of correlations in the microcanonical ensemble are identical in the thermodynamic limit, but we have no proof.) In $[21,10]$ it is proven that, for all $z>0,0<\beta<\frac{4 \pi}{R^{2}}$ and all $n, m$, the thermodynamic limit of the correlation functions of $n$ vortices of vorticity $+R$ at positions $\xi^{n}$ and $m$ vortices of vorticity $-R$ at positions $\bar{\xi}^{m}$,

$$
\varrho^{X}\left(\beta, z ; \xi^{n}, \bar{\xi}^{m}\right)=\lim _{\Lambda \rightarrow \mathbb{R}^{2}} \varrho_{\Lambda}^{X}\left(\beta, z ; \xi^{n}, \bar{\xi}^{m}\right)
$$

exists if $\Lambda \succ \mathbb{R}^{2}$ by inclusion, and for $X=D, F$, and $N$. (For the definition of the grand canonical correlation functions see $[25,10]$.) The limiting functions, $\varrho^{X}$, are Euclidean invariant. Of particular interest are the correlations of the vorticity, $\omega(x)$, which we denote by $\left\langle\omega\left(\xi_{1}\right) \ldots \omega\left(\xi_{n}\right)\right\rangle^{X}(\beta, z)$. (This is the expectation value of the product of the vorticity at $\xi_{1}, \ldots, \xi_{n}$.) It is well known (see e.g. [11]) that

$$
\langle\omega(\xi) \omega(\eta)\rangle^{X}(\beta, z)=\varrho^{X}(\beta, z ; 0) \delta(\xi-\eta)-2\left[\varrho^{X,+-}(\beta, z ; \xi, \eta)-\varrho^{X,++}(\beta, z ; \xi, \eta)\right]
$$

Here $\varrho^{X}(\beta, z ; \xi)$ is the one-vortex correlation which is constant in $\xi$, and the superscripts on the right side of (3.34) indicate the sign of the vorticity of the two vortices.

It follows from $[11,8,9]$ that $\varrho^{X,+-}(\beta, z ; \xi, \eta)-\varrho^{X,++}(\beta, z ; \xi, \eta)$ is a positive, convex function of $|\xi-\eta|$ which tends to 0 , as $|\xi-\eta| \rightarrow \infty$, for $X=D, F$, and $N$. Thus

$$
\langle\omega(\xi) \omega(\eta)\rangle^{X}(\beta, z), \quad \xi \neq \eta
$$

is a negative, concave function of $|\xi-\eta|$ which increases to 0 , as $|\xi-\eta| \rightarrow \infty$. This means that if the vorticity at the point $\xi$ is constrained to be positive, it is predominantly negative at all points $\eta \neq \xi$ (in contrast to what might be expected heuristically). For $X=P$, one can still show that $\langle\omega(\xi) \omega(\eta)\rangle_{\Lambda}^{P}(\beta, z)$ is negative, for $\xi \neq \eta$, in any bounded rectangle $\Lambda$.

Much less is known about systems of vortices of negative vorticity (for example, $-R$ ), immersed in a neutralizing positive background vorticity. This system is stable for arbitrary values of the inverse temperature $\beta$ - in contrast to the two-component vortex plasma. The thermodynamic limits of the free energy and the pressure have been constructed [26], and results similar to (3.22), (3.26), and (3.27) can be derived. However, the microcanonical ensemble does not seem to have been analyzed directly, and the existence of correlation functions is only known for one special value of $\beta$, [14] (see also [7]). It is an interesting speculation that for large values of the inverse temperature $\beta$ the correlation functions of this system exhibit directional long range order. We do however not have a proof of this. 


\section{Properties of the Entropy}

In Sect. 1 we have shown that

$$
V_{\Lambda, D} \leqq\left(\begin{array}{l}
V_{\Lambda, F} \\
V_{\Lambda, P}
\end{array}\right) \leqq V_{\Lambda, N},
$$

in the sense of quadratic forms; see also [24, 23]. From these inequalities and the definition of the kinetic energy, $K$, in Sect. 2 [see (2.1) and (3.1)] we conclude that

$$
U_{\Lambda, D}\left(\xi^{n}, \bar{\xi}^{m}\right) \leqq\left(\begin{array}{l}
U_{A, F}\left(\xi^{n}, \bar{\xi}^{m}\right) \\
U_{\Lambda, P}\left(\xi^{n}, \bar{\xi}^{m}\right)
\end{array}\right) \leqq U_{\Lambda, N}\left(\xi^{n}, \bar{\xi}^{m}\right)
$$

for arbitrary $\xi^{n} \in \Lambda^{n}, \bar{\xi}^{m} \in \Lambda^{m}$. Now, recall the definitions of $\Omega(\Lambda, n, E)$ and of $s_{\Lambda}(\varrho, \varepsilon)$ - see (3.2') and (3.3). Since $\delta^{-}\left(U_{\Lambda, X}\left(\xi^{n}, \bar{\xi}^{n}\right)-E\right)$ is monotone decreasing in $U_{\Lambda, X}$, inequalities (4.2) give

$$
s_{\Lambda}^{N}(\varrho, \varepsilon) \leqq\left(\begin{array}{c}
s_{\Lambda}^{F}(\varrho, \varepsilon) \\
s_{\Lambda}^{P}(\varrho, \varepsilon)
\end{array}\right) \leqq s_{\Lambda}^{D}(\varrho, \varepsilon) .
$$

This proves the first two inequalities in (3.7) and inequality (3.32).

Next, we show that the thermodynamic limit of $s_{\Lambda}^{N}(\varrho, \varepsilon)$ exists. Let $\Lambda$ be the union of $m$ sets $\Delta_{i}, i=1, \ldots, m$, with disjoint interiors. By (1.8)

$$
V_{\Lambda, N} \leqq \sum_{i=1}^{m} V_{\Delta_{i}, N}
$$

in the sense of quadratic forms on functions $f(\xi)$ with the property that $\int f(\xi) d^{2} \xi=0$, for all $i$. [If $f$ violates this condition, for some $i$, we set $\left(f, V_{\Delta_{i}, N} f\right)$ $\stackrel{\Delta_{i}}{=}+\infty ;(4.4)$ then holds in general.] Let $\xi_{\Delta_{t}}$ be the subset of points of $\xi^{n}$ contained in $\Delta_{i}$, and $\bar{\xi}_{\Delta_{t}}$ the subset of $\bar{\xi}^{n}$ contained in $\Delta_{i}$. By (4.4) and (3.1),

$$
U_{\Lambda, N}\left(\xi^{n}, \bar{\xi}^{n}\right) \leqq \sum_{i} U_{\Delta_{i}, N}\left(\xi_{\Delta_{i}}, \bar{\xi}_{\Delta_{\imath}}\right) \text {. }
$$

Since $\delta^{-}$is monotone decreasing in $U$, we conclude that

$$
\Omega^{N}(\Lambda, n, E) \geqq\left(\frac{1}{n !}\right)^{2} \int_{\Lambda^{2 n}} \delta^{-}\left(\sum_{i} U_{\Delta_{i}, N}\left(\xi_{\Delta_{i}}, \bar{\xi}_{\Delta_{i}}\right)-E\right) \cdot d \xi^{n} d \bar{\xi}^{n}
$$

We set $\lambda_{i} \equiv \frac{\left|\Delta_{i}\right|}{|\Lambda|}$. Clearly, $0<\lambda_{i}<1$ and $\sum \lambda_{i}=1$. We choose $n$ and $\left|\Delta_{i}\right|, i=1, \ldots, m$, such that $k_{i}=\lambda_{i} n$ are integers, for all $i$. Finally we set $E_{i} \equiv \lambda_{i} E$. By (4.6),

$$
\Omega^{N}(\Lambda, n, E) \geqq \prod_{i=1}^{m}\left\{\left(\frac{1}{k_{i} !}\right)^{2} \int_{\left(\Delta_{i}\right)^{2 k_{i}}} \delta^{-}\left(U_{\Delta_{i}, N}\left(\xi^{k_{2}}, \bar{\xi}^{k_{i}}\right)-E_{i}\right) \cdot d \xi^{k_{i}} d \bar{\xi}^{k_{1}}\right\} .
$$

By taking logarithms we find

$$
S_{\Lambda}^{N}(\varrho, \varepsilon) \geqq \sum_{i=1}^{m} \lambda_{i} s_{\Delta_{i}}^{N}(\varrho, \varepsilon) .
$$


Since $\delta^{-} \leqq 1$,

$$
\Omega^{N}(\Lambda, n, E) \leqq \frac{|\Lambda|^{2 n}}{(n !)^{2}}, \quad \text { i.e. } \quad s_{\Lambda}^{N}(\varrho, \varepsilon) \leqq \varrho\left(1-\log \frac{\varrho}{2}\right) .
$$

By standard arguments [25], (4.7) and (4.8) imply the existence of the thermodynamic limit of $s^{N}$.

Next, we compare the entropy with the free energy. Since

$$
\delta^{-}(x-E) \leqq e^{-\beta(x-E)},
$$

for all $\beta$, we obtain from $\left(3.2^{\prime}\right)$ and (3.10)

$$
\Omega^{X}(\Lambda, n, E) \leqq Q^{X}(\Lambda, n, \beta) e^{\beta E},
$$

hence

$$
s_{\Lambda}^{\delta, X}(\varrho, \varepsilon) \leqq s_{\Lambda}^{X}(\varrho, \varepsilon) \leqq \beta \varepsilon-\beta f_{\Lambda}^{X}(\varrho, \beta) .
$$

As shown in $[8,9]$ and Appendix $\mathrm{B}, f_{A}^{X}(\varrho, \beta)$ is bounded uniformly in $\Lambda$, for arbitrary $\varrho$ and $X=D, F, P, N$, provided

Conversely,

$$
\beta<4 \pi R^{-2} .
$$

where

$$
Q^{X}(\Lambda, n, \beta) \leqq \sum_{j=1}^{l} Q_{j}+Q_{<}+Q_{>},
$$

$$
\begin{aligned}
Q_{j} & \equiv\left(\frac{1}{n !}\right)^{2} \int_{\Lambda^{2 n}} \exp \left[-\beta U_{\Lambda, X}\left(\xi^{n}, \bar{\xi}^{n}\right)\right] \delta^{E_{j+1}-E_{j}}\left(U_{A, X}\left(\xi^{n}, \bar{\xi}^{n}\right)-E_{j+1}\right) d \xi^{n} d \bar{\xi}^{n} \\
& \leqq \exp |\Lambda|\left\{-\beta \varepsilon_{j+1}+s_{\Lambda}^{\delta}\left(\varrho, \varepsilon_{j+1}\right)\right\}
\end{aligned}
$$

where

$$
\begin{aligned}
\varepsilon_{j} & \equiv \frac{E_{j}}{|\Lambda|}, \quad \delta_{j} \equiv|\Lambda|^{-1}\left(E_{j+1}-E_{j}\right) \equiv \delta=\mathrm{const} ; \\
Q_{<} & \equiv\left(\frac{1}{n !}\right)^{2} \int_{\lambda^{2 n}} e^{-\beta U_{\Lambda, X}\left(\xi^{n}, \bar{\xi}^{n}\right)} \delta^{-}\left(U_{\Lambda, X}\left(\xi^{n}, \bar{\xi}^{n}\right)-E_{1}\right) d \xi^{n} d \bar{\xi}^{n} \\
& \leqq Q^{X}(\Lambda, n, \beta+\gamma) e^{\gamma E_{1}},
\end{aligned}
$$

and we have used (4.9). Given $\beta<4 \pi R^{-2}$, we choose $\gamma>0$ so small that $\beta+\gamma$ $<4 \pi R^{-2}$. Then $Q^{X}(\Lambda, n, \beta+\gamma) \leqq$ const $^{|\Lambda|}$, independent of our choice of $E_{1}$ (see $[8,9]$ and Appendix B). Finally

$$
\begin{aligned}
Q_{>} & \equiv\left(\frac{1}{n !}\right)^{2} \int_{\Lambda^{2 n}} e^{-\beta U_{\Lambda, X}\left(\xi^{n}, \bar{\xi}^{n}\right)} \delta^{-}\left(E_{l}-U_{l, X}\left(\xi^{n}, \bar{\xi}^{n}\right)\right) d \xi^{n} d \bar{\xi}^{n} \\
& \leqq e^{-\beta E_{l}} \frac{|\Lambda|^{2 n}}{(n !)^{2}} .
\end{aligned}
$$

These estimates permit us to use the arguments in [25, Sect. 3.4.3], to conclude that

$$
-\beta f_{\Lambda}^{X}(\varrho, \beta) \leqq \max _{\varepsilon}\left(s_{\Lambda}^{\delta, X}(\varrho, \varepsilon)-\beta \varepsilon\right)+\mathscr{E}_{A},
$$


with $\mathscr{E}_{\Lambda} \rightarrow 0$, as $|\Lambda| \rightarrow \infty$, if (for each $\Lambda$ ) $l, E_{1}$, and $E_{l}$ are chosen suitably. Combining (4.10) and (4.11) we obtain

$$
\beta f^{X}(\varrho, \beta)=\min _{\varepsilon}\left(\beta \varepsilon-\varliminf_{\Lambda>\mathbb{R}^{2}} s_{\Lambda}^{\delta, X}(\varrho, \varepsilon)\right),
$$

where $f^{X}(\varrho, \beta)=\lim _{\Lambda>\mathbb{R}^{2}} f_{\Lambda}^{X}(\varrho, \beta)$, with $\Lambda \succ \mathbb{R}^{2}$, e.g. in the sense of van Hove [25]; see Sect. 5.

Next, we exploit the scaling properties of the Green's function $V_{\Lambda, X}$ of the Laplacian with $X$ b.c. at $\partial \Lambda$ and of the one-body potential $W_{A, X}$, in order to establish the scaling properties of the entropy [see (3.8) and (3.9), Sect. 3.2]. Let $\theta$ be an arbitrary, positive number. We set

$$
\theta^{-1} \Lambda=\{\xi: \theta \xi \in \Lambda\} .
$$

Lemma 4.1. For $X=D, P, N$,

1) $V_{\Lambda, X}(\theta \xi, \theta \eta)=V_{\theta^{-1} \Lambda, X}(\xi, \eta)$.

2) $W_{\Lambda, X}(\theta \xi)=W_{\theta^{-1} \Lambda, X}(\xi)+(1 / 4 \pi) \log \theta$.

For all choices of $X$

3) $U_{\Lambda, X}\left((\theta \xi)^{n},(\theta \bar{\xi})^{m}\right)=U_{\theta^{-1} \Lambda, X}\left(\xi^{n}, \bar{\xi}^{m}\right)+\frac{(n+m) R^{2}}{4 \pi} \log \theta$.

Proof. 1) Let $X=D, P$ or $N$. The Green's function $V_{A, X}(\xi, \eta)$ is uniquely specified by the following properties:

a) For $\eta \in \Lambda, V_{A, X}(\xi, \eta)$ is harmonic in $\xi$ in $\Lambda \sim\{\eta\} ; V_{A, X}(\xi, \eta)=V_{A, X}(\eta, \xi)$.

b) For $\eta \in \Lambda, V_{\Lambda, X}(\xi, \eta) \approx-\frac{1}{2 \pi} \log |\xi-\eta|+$ const, as $\xi \rightarrow \eta$.

$\left.\mathrm{c}_{\mathrm{D}}\right) V_{\Lambda, D}(\xi, \eta) \rightarrow 0$, if either $\xi$ or $\eta$ approach $\partial \Lambda$.

$\left.\mathrm{c}_{\mathrm{N}}\right)\left(\frac{\partial}{\partial n_{\xi}} V_{\Lambda, N}\right)(\xi, \eta)=0$, for $\xi \in \partial \Lambda$.

$\left.\mathrm{c}_{\mathrm{P}}\right) V_{\Lambda, P}(\xi, \eta)$ is periodic in $\xi$ and $\eta$, with domain of periodicity $=\Lambda$.

Now, note that $V_{A, X}(\theta \xi, \theta \eta)$ satisfies a), b), and $\left.\mathrm{c}_{\mathrm{X}}\right)(X=D, P, N)$ if in a), b), and $\left.\mathrm{c}_{\mathrm{X}}\right) \Lambda$ is replaced by $\theta^{-1} \Lambda$. This proves 1$)$. Lemma 4.1,2) then follows from (1.3), (1.4), and (1.5). Using 1) and 2) we finally see that 3) follows from (3.1) and (1.3).

We are now prepared to prove the scaling relation (3.8) for the entropy $s_{\Lambda}^{X}(\varrho, \varepsilon)$. (We temporarily suppress the super- and subscripts $X$.) By (3.2') and (3.3), Sect. 3.1,

$$
s_{\Lambda}(\varrho, \varepsilon)=\frac{1}{|\Lambda|} \log \left\{\left(\frac{1}{n !}\right)^{2} \int_{\Lambda^{2 n}} \delta^{-}\left(U_{\Lambda}\left(\xi^{n}, \bar{\xi}^{n}\right)-E\right) d \xi^{n} d \bar{\xi}^{n}\right\},
$$

with $\varrho \equiv \frac{2 n}{|\Lambda|}, \varepsilon \equiv \frac{E}{|\Lambda|}$. We now make a change of variables, $\xi_{j} \rightarrow \eta_{j}=\theta^{-1} \xi_{j}, \bar{\xi}_{j} \rightarrow \bar{\eta}_{j}$ $=\theta^{-1} \bar{\xi}_{j}, j=1, \ldots, n$. We set

$$
(\theta \eta)^{n}=\left(\theta \eta_{1}, \ldots, \theta \eta_{n}\right), \quad \text { etc. }
$$

Note that $\eta^{n}$ and $\bar{\eta}^{n}$ range over $\left(\theta^{-1} \Lambda\right)^{n}$, and

$$
d \xi^{n}=\theta^{2 n} d \eta^{n}, \text { etc. }
$$


Hence

$$
\begin{aligned}
s_{\Lambda}(\varrho, \varepsilon)= & \theta^{-2} \frac{1}{\left|\theta^{-1} \Lambda\right|} \\
& \cdot \log \left\{\left(\frac{1}{n !}\right)^{2} \theta^{4 n} \int_{\left(\theta^{-1} \Lambda\right)^{2 n}} \delta^{-}\left(U_{\theta^{-1} \Lambda}\left(\eta^{n}, \bar{\eta}^{n}\right)+\frac{2 n R^{2}}{4 \pi} \log \theta-E\right) d \eta^{n} d \bar{\eta}^{n}\right\},
\end{aligned}
$$

i.e.

$$
s_{\Lambda}(\varrho, \varepsilon)=\theta^{-2} s_{\theta^{-1} \Lambda}\left(\theta^{2} \varrho, \theta^{2} \varepsilon+\frac{\varrho R^{2}}{4 \pi} \theta^{2} \log \theta\right)+2 \varrho \log \theta,
$$

which is (3.8).

\section{Free Energy and Pressure; Conclusions}

\subsection{Existence of the Thermodynamic Limit}

Combining definitions (3.10), (3.11) with inequality (4.2) we obtain

$$
f_{\Lambda}^{D}(\varrho, \beta) \leqq\left(\begin{array}{l}
f_{\Lambda}^{F}(\varrho, \beta) \\
f_{\Lambda}^{P}(\varrho, \beta)
\end{array}\right) \leqq f_{\Lambda}^{N}(\varrho, \beta)
$$

and, using in addition (3.14), we get

$$
0<p_{\Lambda}^{N}(z, \beta) \leqq\left(\begin{array}{l}
p_{\Lambda}^{F}(z, \beta) \\
p_{\Lambda}^{D}(z, \beta)
\end{array}\right) \leqq p_{\Lambda}^{D}(z, \beta) .
$$

In Appendix $\mathrm{B}$ it is shown that, for all $z$ and all $\beta<4 \pi R^{2}$,

$$
p_{\Lambda}^{D}(z, \beta) \leqq \text { const }, \quad \Lambda \supseteqq\{\xi:|\xi| \leqq 1\},
$$

for some finite constant independent of $\Lambda$. Inequalities (5.1) and (5.2) yield (3.16).

By (3.10), (4.4), and (4.5)

$$
Q^{N}(\Lambda, n, \beta) \geqq \prod_{i=1}^{m} Q\left(\Delta_{i}, k_{i}, \beta\right)
$$

i.e.

$$
f_{\Lambda}^{N}(\varrho, \beta) \leqq \sum_{i=1}^{m} \lambda_{i} f_{\Delta_{i}}^{N}(\varrho, \beta)
$$

where $\lambda_{i} \equiv\left|\Delta_{i}\right| /|\Lambda|, k_{i}=\lambda_{i} n, \ldots$, as in Sect. 4 . If $\Lambda$ is a unit square, for example, then clearly

$$
Q^{N}(\Lambda, k, \beta)>0
$$

for all $k$ and all $\beta<4 \pi / R^{2}$. Furthermore, since $Q^{N}(\Lambda, n, \beta) \leqq Q^{D}(\Lambda, n, \beta) \leqq$ const $^{n}$, if $\beta<4 \pi / R^{2}$ and $2 n /|\Lambda|$ is bounded uniformly in $\Lambda$ (see Appendix B), we conclude that $f_{A}^{N}(\varrho, \beta)$ satisfies uniform upper and lower bounds, for all $\varrho>0$ and all $\beta<4 \pi / R^{2}$. This and (5.3) show that if $\Lambda \nearrow \mathbb{R}^{2}$, in the sense of van Hove [25],

$$
\lim _{\Lambda>\mathbb{R}^{2}} f_{\Lambda}^{N}(\varrho, \beta) \equiv f^{N}(\varrho, \beta)
$$


exists. An argument originally due to Griffiths [12] can be used to show that the thermodynamic limit of $f_{A}^{F}(\varrho, \beta)$ exists. See also $[8,9,13]$. For $X=D, P$ we do not give a direct proof of the existence of the thermodynamic limit of the free energy, but analyze the pressure and then exhibit the equivalence of the canonical and the grand-canonical ensembles (Sect. 5.2).

The existence of the thermodynamic limit of the pressure for periodic b.c. can be inferred from $[9,6]$. (The arguments are somewhat lengthy and are therefore not reproduced here.) For $X=D, F$, and $N$, we can establish the existence of the thermodynamic limit of the pressure by considering its first derivative in $z$ :

$$
z \frac{\partial\left(\beta p_{\Lambda}^{X}(z, \beta)\right)}{\partial z}=|\Lambda|^{-1} \int_{\Lambda} d^{2} \xi \varrho_{\Lambda}^{X}(\beta, z ; \xi)
$$

i.e.

$$
\beta p_{\Lambda}^{X}(z, \beta)=|\Lambda|^{-1} \int_{0}^{z} d \zeta \zeta^{-1} \int_{\Lambda} d^{2} \xi \varrho_{\Lambda}^{X}(\beta, \zeta ; \xi) .
$$

By correlation inequalities $[10] \varrho_{A}^{X}(\beta, z ; \xi)$ is increasing in $\Lambda$, for $X=F, N$, while $\varrho_{\Lambda}^{D}(\beta, z ; \xi)$ is decreasing in $\Lambda$, for each fixed $\xi$. These properties along with (5.4) and (5.1), (5.2) establish (3.17) and the existence of the thermodynamic limit. (For $X=F, N$ one could instead use Griffiths' argument [12].)

Remark. The correlation inequalities in $[10,21]$ can be used to construct the thermodynamic limit of all grand-canonical correlation functions.

\subsection{Scaling Properties of the Pressure and Free Energy}

We recall the definition (3.10) of the canonical partition function. (We temporarily suppress reference to boundary conditions.)

$$
Q(\Lambda, n, m, \beta)=\frac{1}{n ! m !} \int_{\Lambda^{n+m}} d \xi^{n} d \bar{\xi}^{m} e^{-\beta U_{\Lambda}\left(\bar{\xi}^{n}, \bar{\xi}^{m}\right)} .
$$

We change variables, $\xi_{j} \rightarrow \eta_{j} \equiv \theta^{-1} \xi_{j}, \ldots$, as in Sect. 4. By Lemma 4.1,

$$
\begin{aligned}
Q(\Lambda, n, m, \beta)= & \frac{\theta^{2(n+m)}}{n ! m !} \int_{\left(\theta^{-1} \Lambda\right)^{n+m}} d \eta^{n} d \bar{\eta}^{m} \\
& \cdot \exp \left[-\beta U_{\theta^{-1} \Lambda}\left(\eta^{n}, \bar{\eta}^{m}\right)\right] \exp \left[-\beta(n+m) \frac{R^{2}}{4 \pi} \log \theta\right] \\
= & \theta^{(n+m)\left(2-\beta R^{2} / 4 \pi\right)} Q\left(\theta^{-1} \Lambda, n, m, \beta\right) .
\end{aligned}
$$

With (3.14) this yields

$$
\Xi\left(\Lambda, z^{\prime}, \beta\right)=\Xi\left(\theta^{-1} \Lambda, \theta^{2-\beta R^{2} / 4 \pi} z^{\prime}, \beta\right),
$$

hence

$$
p_{\Lambda}\left(z^{\prime}, \beta\right)=\theta^{-2} p_{\theta^{-1} \Lambda}\left(\theta^{2-\alpha} z^{\prime}, \beta\right),
$$

where $\alpha \equiv \beta R^{2} / 4 \pi$. Now choose $z^{\prime}=1, z=\theta^{2-\alpha}$. After passing to the thermodynamic limit and setting $\beta p(1, \beta) \equiv F(\alpha)$, we obtain $\beta p(z, \beta)=z^{2 /(2-\alpha)} F(\alpha)$, which proves Eq. (3.27), Theorem 3.2. Moreover, it shows that $p(z, \beta)$ is analytic in $z$, 
except at $z=0, \infty$, and strictly convex on the positive real axis, for arbitrary $\alpha \in(0,1)$. This proves the equivalence of the canonical and grand canonical ensembles. Therefore the thermodynamic limit of $f_{A}^{X}$ exists for all $X=D, F, P, N$, and $f^{X}$ satisfies Eq. (3.28), Theorem 3.2.

\subsection{Comments on the Equivalence}

of the Canonical and Microcanonical Ensembles; Conclusions

Notice that if we can prove that the function $F^{X}(\alpha)$ is continuously differentiable in $\alpha$ at some value $\alpha_{0}$ of $\alpha$, then $\beta f^{X}(\beta, \varrho)$ is continuously differentiable in $\beta$ at $\beta_{0}=\frac{4 \pi \alpha_{0}}{R^{2}}$. In that case

$$
\left.s^{\delta, X}(\varrho, \varepsilon)=s^{X}(\varrho, \varepsilon)=\beta_{0} \varepsilon-\beta_{0} f^{X}\left(\varrho, \beta_{0}\right), \quad \varepsilon=-\frac{\partial}{\partial \beta}\left(\beta f^{X}(\varrho, \beta)\right)\right)_{\beta=\beta_{0}},
$$

see e.g. [25]. If continuous differentiability is true for all $\beta_{0} \in\left(0,4 \pi / R^{2}\right)$, it follows that the thermodynamic limit $s^{\delta, X}(\varrho, \varepsilon)$ of the entropy exists for all $\varrho>0$ and all real $\varepsilon$ (see Theorem 3.2), and $s^{\delta, X}(\varrho, \varepsilon)$ would be a strictly increasing function of $\varepsilon$.

We can think of two techniques that might enable one to derive differentiability properties of $F^{X}(\alpha)$ :

1) One could try to extend the techniques of Brydges and Federbush $[4,5]$ to the two-dimensional continuum Coulomb gas. This would enable one to prove that for $0<\alpha<\tilde{\alpha}$, for some $\tilde{\alpha}<1, F^{D}(\alpha)$ is $C^{\infty}$ in $\alpha$ and to determine the rate of divergence of $\frac{d}{d \alpha} F^{D}(\alpha)$, as $\alpha \rightarrow 0$ : In the thermodynamic limit

$$
\frac{\partial\left(\beta p^{D}(z, \beta)\right)}{\partial \beta}=-\frac{R^{2}}{2 \pi} \int d^{2} \xi \ln |\xi|\left[\varrho^{+-}(\beta, z ; \xi, 0)-\varrho^{++}(\xi, z ; \xi, 0)\right] .
$$

Taking for granted that the methods of $[4,5]$ apply to the continuum gas, one would conclude that

$$
\varrho^{+-}(\beta, z ; \xi, 0)-\varrho^{++}(\beta, z ; \xi, 0) \approx \operatorname{const} \beta \int d^{2} k \frac{e^{i k \xi}}{k^{2}+m(\beta)^{2}},
$$

and the inverse correlation length, $m(\beta)$, behaves like

$$
m(\beta)^{2} \approx 2 z \beta,
$$

as $\beta \rightarrow 0$, up to corrections of higher order in $\beta$. (These asymptotic formulas are suggested by Debye-Hückel theory.) By inserting these results in (5.6) we conclude that

$$
\frac{\partial\left(\beta p^{D}(z, \beta)\right)}{\partial \beta} \approx \text { const } \ln \beta,
$$

as $\beta \rightarrow 0$.

2) One can try to exploit the results of Faddeev et al. [27], claimed to be exact results for the two-dimensional sine-Gordon theory. That theory is isomorphic to the two-dimensional, two-component Coulomb gas studied here $[8,9]$. The vacuum energy density of the sine-Gordon theory, normalized such that it 
vanishes in the free-field limit, i.e. when $\beta \rightarrow 0$ or $z \rightarrow 0$, is precisely the pressure of the Coulomb gas. The results in [27] indicate that the pressure is a smooth function of $\beta$ in the interval $\left(0,4 \pi / R^{2}\right)$. This would imply that the function $F(\alpha)$, see Theorem 3.2, is smooth in $\alpha \in(0,1)$, and therefore the entropy is uniquely determined by the free energy.

\subsection{Reduction to a Box of Fixed Size}

We have discussed the situation where $2 n$ vortices with fixed strength $\pm R$ are contained in a box $\Lambda \rightarrow \infty$, with $n /|\Lambda|$ constant. Because of the scaling properties of the electrostatic potential, it is equivalent to consider $2 n$ vortices of strength $\pm R$ in a fixed box $\Lambda$. We might also take $2 n$ vortices of strength $\pm R_{n} \rightarrow 0$ in a fixed box $\Lambda$. There is some arbitrariness in the choice of the energy variable, and different choices may lead to different limiting regimes. It is not a priori clear which one is appropriate for the statistical mechanics of vortices (as Oscar Lanford kindly pointed out to us). The limiting regime which we studied gave the entropy as a nontrivial function of the energy (continuous nonconstant function). Essentially different limiting regimes would thus presumably lead to trivial functions. In particular, we don't see how to obtain an entropy function which would increase on one interval and decrease in another interval as suggested by the Onsager argument.

\section{Appendix A}

Proof of Theorem 3.1. We know that the limits

$$
f^{X}(\varrho, \beta)=\beta^{-1} \lim |\Lambda|^{-1} \log \left\{\frac{1}{(n !)^{2}} \int_{A^{2 n}} d \xi^{n} d \bar{\xi}^{n} e^{-\beta U_{A}^{X}\left(\xi^{n}, \bar{\xi}^{n}\right)}\right\}
$$

exist when $\Lambda>\mathbb{R}^{2}$, in the sense of van Hove, and $|\Lambda|^{-1} 2 n \rightarrow \varrho$, defining the free energy density, for the boundary conditions $X=D, F, P, N$. We have shown that $f^{X}(\varrho, \beta)=-\infty$, for $\beta \geqq \frac{4 \pi}{R^{2}}$, and $\beta f^{X}(\varrho, \beta)$ is a finite concave function of $\beta$ on $\left(0, \frac{4 \pi}{R^{2}}\right)$, with $f^{X}(\varrho, \beta) \rightarrow-\infty$ when $\beta \rightarrow \frac{4 \pi}{R^{2}}$. This is part of the content of Theorem 3.2, and is proved without the help of the present appendix. Define

$$
\sigma^{X}(\varrho, \varepsilon)=\inf _{\beta \in\left(0, \frac{4 \pi}{R^{2}}\right)}\left(\beta \varepsilon-\beta f^{X}(\varrho, \beta)\right) .
$$

This is clearly an increasing function of $\varepsilon$. Let $\varepsilon_{L}(\beta)$ and $\varepsilon_{R}(\beta)$ be the left and right derivative of $\beta f^{X}(\varrho, \beta)$ with respect to $\beta$. We shall from now on omit the superscript $X$. General arguments concerning the equivalence of ensembles [25] give the following results:

(a) $\lim _{\Lambda>\mathbb{R}^{2}} s_{\Lambda}^{\delta}(\varrho, \varepsilon)=\lim _{\Lambda>\mathbb{R}^{2}} s_{\Lambda}(\varrho, \varepsilon)=\sigma(\varrho, \varepsilon)=\beta \varepsilon-\beta f(\varrho, \varepsilon)$

when $\varepsilon=\varepsilon_{R}(\beta)$. 
(b) $\limsup _{\boldsymbol{\Lambda} \rightarrow \mathbb{R}^{2}} s_{\Lambda}^{\delta}(\varrho, \varepsilon) \leqq \sigma(\varrho, \varepsilon)$

when $\varepsilon \in\left[\varepsilon_{R}(\beta), \varepsilon_{L}(\beta)\right]\left(\sigma(\varrho, \cdot)\right.$ is then linear on $\left.\left[\varepsilon_{R}(\beta), \varepsilon_{L}(\beta)\right]\right)$.

Proposition. For all $X$ and $\varepsilon>\varepsilon_{R}(\beta)$,

$$
\liminf _{\Lambda>\mathbb{R}^{2}} s_{\Lambda}^{\delta}(\varrho, \varepsilon) \geqq \sigma\left(\varrho, \varepsilon_{R}(\beta)\right)
$$

when $\Lambda>\mathbb{R}^{2}$ in the sense of Fisher ${ }^{9}$.

We shall prove this proposition for $X=D$ which is the most difficult, but also the most interesting case. Other b.c. can be analyzed in a very similar way.

We now establish some notation:

$$
\varepsilon_{0} \equiv \varepsilon_{R}(\beta), \quad s_{0}=\sigma\left(\varrho, \varepsilon_{R}(\beta)\right) .
$$

Instead of specifying points $\left(\xi^{n}, \bar{\xi}^{n}\right)$ in $\Lambda^{2 n}$, the positions of vortices, we shall specify configurations, $X$, a set of $n+$ vortices and a set of $n-$ vortices. If $S$ is a set of configurations, $X$, and $\tilde{S}$ the corresponding subset of $\Lambda^{2 n}$ [i.e. $\left.\tilde{S}=\left\{\left(\xi^{n}(X), \bar{\xi}^{n}(X)\right): X \in S\right\}\right]$, we define

$$
\operatorname{vol}_{n} S=\frac{1}{(n !)^{2}} \int d \xi^{n} d \bar{\xi}^{n} \chi_{\tilde{S}}\left(\xi^{n}, \bar{\xi}^{n}\right),
$$

with $\chi_{\tilde{S}}$ the characteristic function of $\tilde{S}$.

Given an arbitrary $\gamma>0$ and $\varepsilon>\varepsilon_{0}$, we shall try, for large $\Lambda$ and $|\Lambda|^{-1} 2 n \approx \varrho$, to construct a set $S$ of configurations such that

$$
|\Lambda|^{-1} \log \operatorname{vol}_{n} S>s_{0}-\gamma,
$$

and such that all configurations in $S$ have energy in the interval $((\varepsilon-\delta)|\Lambda|,(\varepsilon+\delta)|\Lambda|)$, for some arbitrarily small, but positive $\delta$. Since $\varepsilon>\varepsilon_{0}$ and by (A.2), this proves the proposition.

The proof of (A.3) consists of an explicit construction of $S$ which we now outline: We choose an integer $v \ll n$, with $v \rightarrow \infty$ and $v / n \rightarrow 0$, as $n \rightarrow \infty$, and consider configurations of $2(n-v)$ vortices in $\Lambda$ with energy in an interval $\left[\left(\varepsilon_{0}-\delta^{\prime}\right)|\Lambda|, \varepsilon_{0}|\Lambda|\right]$. To these configurations we add the $2 v$ remaining vortices in a small number (two) of very concentrated clusters in such a way that they contribute an amount of energy proportional to $|\Lambda|$ and make a negligible contribution to the specific entropy.

Let $S_{0}$ be the set of configurations of $2(n-v)$ vortices with energy in the interval $\left[\left(\varepsilon_{0}-\delta^{\prime}\right)|\Lambda|, \varepsilon_{0}|\Lambda|\right]$. Since $v / n \rightarrow 0$, we have for large $\Lambda$,

$$
|\Lambda|^{-1} \log \operatorname{vol}_{n-v} S_{0}>s_{0}-\gamma / 3 .
$$

We may also assume that $\gamma$ has been chosen so small that

$$
|\Lambda|^{-1} \log \operatorname{vol}_{n} S_{0}^{<}<s_{0}-2 \gamma,
$$

where $S_{0}^{<}$is the set of configurations with energy $<\left(\varepsilon_{0}-\delta^{\prime}\right)|\Lambda|$. Since $\Lambda \succ \mathbb{R}^{2}$ in the sense of Fisher, we may decompose most of the area of $\Lambda$ into little squares, $\Sigma$, of area $1 / 2 \varrho$. Let $\langle(\cdot)\rangle$ denote the expectation value given by the measure $\left(\operatorname{vol}_{n-v} S_{0}\right)^{-1} \operatorname{vol}_{n-v}(\cdot)$ on $S_{0}$. Then, for more than half of the squares $\Sigma$,

$$
\langle\text { number of vortices in } \Sigma\rangle \leqq \frac{2}{3} \text {. }
$$

9 See [25] for the definitions 


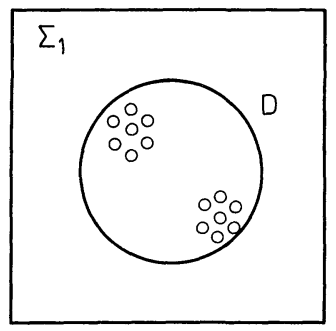

Fig. 2

Since $\Lambda>\mathbb{R}^{2}$ in the sense of Fisher, some fraction (>constant) of these little squares has a distance $\geqq \kappa \operatorname{diam} \Lambda$ to the boundary of $\Lambda$, for some $\kappa>0$. We choose one such square, $\Sigma_{1}$. Let $S_{1} \subset S_{0}$ be the set of all those configurations in $S_{0}$ with no vortices in $\Sigma_{1}$. It follows easily from (A.6) that

$$
\operatorname{vol}_{n-v} S_{1}>\frac{1}{4} \mathrm{vol}_{n-v} S_{0} .
$$

Hence, for $\Lambda$ sufficiently large

$$
|\Lambda|^{-1} \log \operatorname{vol}_{n-v} S_{1}>s_{0}-2 \gamma / 3
$$

an immediate consequence of (A.4) and (A.7). [We note that we could, for each $N=1,2,3, \ldots$, find $N$ squares, $\Sigma_{1}, \ldots, \Sigma_{N}$, at distances $\geqq \kappa \operatorname{diam} \Lambda$ from $\partial \Lambda$, such that the set of configurations $S_{N} \subset S_{0}$ with no vortices in $\Sigma_{1} \cup \ldots \cup \Sigma_{N}$ satisfies $v l_{n-\nu} S_{N}$ $>(1 / 4)^{N} \mathrm{vol}_{n-v} S_{0}$, and $|\Lambda|^{-1} \log \mathrm{vol}_{n-v} S_{N}>S_{0}-2 \gamma / 3$, for sufficiently large $\Lambda$.]

We now modify configurations in $S_{1}$ by adding $v+$ vortices and $v$-vortices in $\Sigma_{1}$. Let $\omega(x)$ denote the charge (or vorticity) density corresponding to the $2 v$ vortices in $\Sigma_{1}$. Clearly

$$
\operatorname{supp} \omega \cong \Sigma_{1}, \quad \int d^{2} x \omega(x)=0, \quad \int d^{2} x|\omega(x)|=2 v R .
$$

As shown in Sect. 1 , it then follows that

$$
\int d^{2} x d^{2} y \omega(x) \omega(y)\left[V_{A, X}(x, y)-V_{\infty}(x-y)\right] \leqq \operatorname{const}(R v)^{2}(\sqrt{2 \varrho} \cdot \kappa \operatorname{diam} \Lambda)^{-1} .
$$

Next, we describe the way in which we distribute the $2 v$ vortices in $\Sigma_{1}$ more precisely: They are all contained in a disc $D$ of radius at most $(1 / 4)(2 \varrho)^{-1 / 2}$ inscribed in $\Sigma_{1}$. Each individual vortex is in a disc of radius $r$,

$$
r \ll\left(\frac{1}{2 \nu \varrho}\right)^{1 / 2}
$$

so that the distance of two such discs is at least $2 r$. The discs containing a positive vortex form a cluster, those containing a negative vortex form another cluster, and the two clusters are at a distance $\approx 1 / 4(2 \varrho)^{-1 / 2}$; see Fig. 2 . To be specific, we may assume that these clusters are roughly circular pieces of a regular lattice (hexagonal or square) of small discs of radius $r$, with lattice distance proportional to $r$. By (A.10), the interaction energy of vortices within one such cluster is

$$
\sim\left(R^{2} v^{2} / 2 \pi\right) \log \left(\frac{1}{r \sqrt{v}}\right)-R^{2} v^{2} O\left(\operatorname{diam} \Lambda^{-1}\right),
$$

$r \sqrt{v} \ll 1$, as $v \rightarrow \infty$. 
The interaction energy between the two different clusters is

$$
\sim-\left(R^{2} v^{2} / 2 \pi\right) \log \left(\frac{1}{4 \sqrt{2 \varrho}}\right)-R^{2} v^{2} O\left(\operatorname{diam} \Lambda^{-1}\right) .
$$

These estimates are to be understood as follows:

(a) Uniform constants only depending on the geometry of the clusters have not been computed.

(b) Apart from those constants, (A.11) and (A.12) give the exact behavior of the total interaction energy of the $2 v$ vortices, in the sense that the interaction energy is contained in an interval

$$
[K-\eta, K] R^{2} v^{2} \log \left(\frac{k}{r \sqrt{v}}\right),
$$

where $K$ and $k$ are constants independent of $r, v$, and $\Lambda$, and $\eta$ tends to 0 , as $r \rightarrow 0$, $\Lambda \rightarrow \infty$.

Next, we must estimate the interaction energy between the $2 v$ vortices in $D \subset \Sigma_{1}$ and the $2(n-v)$ vortices in $\Lambda \backslash \Sigma_{1}$ : We fix a configuration $S_{1}$ of $2(n-v)$ vortices in $\Lambda \backslash \Sigma_{1}$. This configuration determines a charge density, $\Omega(x)$, with supp $\Omega \subset \Lambda \backslash \Sigma_{1}$, and

$$
\int \Omega(x) d^{2} x=0
$$

Next, we fix the position of each of the $2 v$ vortices in $\Sigma_{1}$ to be at the center of one of the little discs of radius $r$ contained in $D \subset \Sigma_{1}$. Let $\omega_{0}(x)$ denote the charge density corresponding to this particular configuration. Furthermore, let $\omega_{\theta}(x)$ denote the charge density obtained from $\omega_{0}(x)$ by rotating the positions of all $2 v$ vortices about the center of $\Sigma_{1}(=$ center of $D)$ through an angle $\theta$.

We now note that

$$
\int_{0}^{2 \pi} d \theta \omega_{\theta}(x) \equiv \bar{\omega}(x)
$$

is invariant under rotations about the center of $\Sigma_{1}$, and $\int \bar{\omega}(x) d^{2} x=0$. The interaction energy between the $2 v$ vortices inside $\Sigma_{1}$ with charge distribution $\omega_{\theta}(x)$ and the $2(n-v)$ vortices of the configurations $S_{1}$ in $\Lambda \backslash \Sigma_{1}$ is given by

$$
W_{\theta} \equiv \int d^{2} x d^{2} y \omega_{\theta}(x) \Omega(y) V_{\Lambda, X}(x, y) .
$$

Next

$$
\int_{0}^{2 \pi} d \theta W_{\theta}=\int d^{2} x d^{2} y \bar{\omega}(x) \Omega(y) V_{\Lambda, X}(x, y) .
$$

Since $V_{A, X}(x, y)$ is a harmonic function of $x$, for $x \in D$, for all choices of b.c. $X$ and all $y \in \Lambda \backslash \Sigma_{1}$, and since $\int \bar{\omega}(x) d^{2} x=0$ it follows that

$$
\int_{0}^{2 \pi} d \theta W_{\theta}=0
$$


As long as $n$ is finite (or, equivalently, $|\Lambda|$ is finite) $W_{\theta}$ is a continuous, periodic function of $\theta$. This is true because

$$
\operatorname{dist}\left(\operatorname{supp} \Omega, \operatorname{supp} \omega_{\theta}\right) \geqq \frac{1}{4}(2 \varrho)^{-1 / 2} .
$$

Thus there exists at least one value $\theta_{S_{1}}$ of $\theta$ such that

$$
W_{\theta_{S_{1}}}=0 \text {. }
$$

As one varies the position of each of the $2 v$ vortices in $D \subset \Sigma_{1}$ throughout one of the small discs of radius $r$ (depicted in Fig. 2), the variation, $\Delta W_{\theta}$, in the interaction energy between the $2 v$ vortices in $D$ and the $2(n-v)$ vortices in $\Lambda \backslash \Sigma_{1}$ is bounded by

$$
\left|\Delta W_{\theta}\right| \leqq \operatorname{const}(v(n-v) / \sqrt{2 \varrho}) \cdot r
$$

This follows from (A.15) and the continuity properties of $V_{\Lambda, X}(x, y)$ with respect to $x \in D$, for arbitrary $y \in \Lambda \backslash \Sigma_{1}$.

Thus if the position of each of the $2 v$ vortices inside $\Sigma_{1}$ is anywhere inside one of the $2 v$ little discs, rotated by $\theta_{S_{1}}$, inside $D$ then, by (A.17) and (A.18),

$$
\left|W_{\theta_{S_{1}}}\right| \leqq \operatorname{const}(v(n-v) / \sqrt{2 \varrho}) \cdot r \text {. }
$$

To complete the proof of our proposition we now must choose $v$ and $r$, calculate the total energy uncertainty, using (A.13) and (A.19), and calculate the entropy of the class of configurations constructed above. For example, we may choose

$$
\left.\begin{array}{l}
v=\left(c_{1}|\Lambda| / \operatorname{diam} \Lambda\right)^{1 / 2}, \\
r=c_{2} v^{-1 / 2} \exp \left[-c_{3} \operatorname{diam} \Lambda\right],
\end{array}\right\}
$$

where $c_{1}, c_{2}$, and $c_{3}$ are finite, positive constants. Then the total energy of the $2 v$ vortices in $D \subset \Sigma_{1}$ is, by (A.13), contained in the interval

$$
c_{4}[K-\eta, K] R^{2}|\Lambda|,
$$

for a positive constant $c_{4}$ (depending smoothly on $c_{1}, c_{2}, c_{3}$ ), with $\eta \rightarrow 0$, as $\Lambda \nearrow \mathbb{R}^{2}$, in the sense of Fisher. Moreover

$$
\left|W_{\theta_{S_{1}}}\right| \leqq c_{5} \sqrt{\varrho}|\Lambda|(|\Lambda| / \operatorname{diam} \Lambda)^{1 / 4} e^{-c_{3} \operatorname{diam} \Lambda} .
$$

Thus the total energy of the configuration, $S$, consisting of $S_{1}$ and of the $2 v$ vortices put into $D$ by the construction described above, is contained in the interval

$$
\left[\left(\varepsilon_{0}+c_{4} K-\delta_{1}\right)|\Lambda|,\left(\varepsilon_{0}+c_{4} K+\delta_{2}\right)|\Lambda|\right]
$$

where

$$
\begin{aligned}
& \delta_{1}=\delta^{\prime}+\eta+\exp [-O(\operatorname{diam} \Lambda)], \\
& \delta_{2}=\exp [-O(\operatorname{diam} \Lambda)] .
\end{aligned}
$$

By choosing $c_{1}, c_{2}, c_{3}$ suitably, $c_{4} K$ can attain any prescribed, positive value. 
To calculate the entropy of $S$, we first calculate the total volume, $V_{v}$, of all configurations of the $2 v$ vortices inside $D$ constructed as described above:

$$
V_{v}=\left(\pi r^{2}\right)^{2 v}=\operatorname{const}\left(\frac{1}{v !}\right) e^{-\operatorname{const} v \operatorname{diam} \Lambda} .
$$

Note that $V_{v}$ is independent of $S_{1}$ (in spite of the fact that the angle $\theta_{S_{1}}$ depends on $S_{1}$ ). Thus the volume of $S$ is given by

$$
\operatorname{vol}_{n} S=\operatorname{vol}_{n-v} S_{1} \cdot V_{v},
$$

and hence, using (A.8), (A.20), and (A.23)

$$
\begin{aligned}
|\Lambda|^{-1} \log \operatorname{vol}_{n} S & =|\Lambda|^{-1} \log \operatorname{vol}_{n-v} S_{1}+|\Lambda|^{-1} \log \left(V_{v}\right) \\
& >s_{0}-2 \gamma / 3-\operatorname{const}\left(|\Lambda|^{-1} \operatorname{diam} \Lambda\right)^{1 / 2} \\
& >s_{0}-\gamma,
\end{aligned}
$$

provided $\Lambda \nearrow \mathbb{R}^{2}$, in the sense of Fisher, and, given $\gamma,|\Lambda|$ is chosen sufficiently large.

Clearly (A.24) and (A.22) complete the proof of (A.3) and hence of our proposition.

Remark. In our proof we have used two special features of the Coulomb interaction:

1) $V_{A, X}(x, y)$ diverges to $+\infty$, as $y \rightarrow x$, for each $x \in D$. [This was used in (A.11) and permitted our choice of $v$, namely $v /|\Lambda|^{1 / 2} \rightarrow 0$, as $|\Lambda| \rightarrow \infty$, see (A.20).]

2) More importantly, in our estimate of the interaction energy, $W$, between the $2 v$ vortices in $D$ and the $2(n-v)$ vortices in $\Lambda \backslash \Sigma_{1}$, we have used the harmonic property of $V_{\Lambda, X}(x, y)$ with respect to $x \in D$, for $y \in \Lambda \backslash \Sigma_{1}$.

It turns out that one can avoid using either of these two elements, 1) and 2). Instead one uses the following elements:

a) Inequality (A.5).

b) Given an arbitrarily small square $\Sigma$, the set of configurations of $v(+$ or -$)$ particles inside $\Sigma$ of energy $\geqq \varepsilon v^{2}$ has a volume $V_{v} \geqq \operatorname{const}(v !)^{-p}, p<\infty$, provided $\varepsilon$ is small enough.

c) Charge conjugation invariance (i.e., + particles and - particles have equal a priori probabilities); or repulsive (positive) two body potentials of short range.

d) One repeats the construction described above in $N$ widely separated squares, $\Sigma_{1}, \ldots, \Sigma_{N}$ [see remark between (A.8) and (A.9)].

These properties are all valid in the vortex gas studied in this paper, but they hold for a much larger class of classical statistical systems. The proof of our Proposition, assuming only a)-c) above, however becomes more difficult. [A clever interplay between d), c), and a) permits us to control the entropy and energy uncertainty.] We do not give the details.

\section{Appendix B}

\section{Stability in the Canonical and Grand Canonical Ensembles, and Monotonicity Properties of the Pressure}

In this appendix we briefly describe two methods for proving the stability bounds (3.12) and (3.16) for $\beta<4 \pi / R^{2}$. In $[8,9]$ these facts have already been established 
for b.c. $X=F, N$. Thanks to the third inequality in (3.16) it suffices to prove the stability bound [last inequality in (3.16)] for Dirichlet b.c. The bound (3.12) follows from (3.16) by standard arguments; see e.g. Corollary 3.6 of [8].

We then briefly indicate how one proves the monotonicity properties (3.17) and, finally, how one can treat vortex gases, where the vorticity, $R$, of individual vortices varies, but is distributed according to some finite measure $d \lambda(R)$ of compact support.

Let $V_{\Lambda, X}^{(\mu)}$ be the Green's function of the operator $-\Delta_{\Lambda, X}+\mu^{2}$, where $\Delta_{\Lambda, X}$ is the Laplacian on $L^{2}$ ( $\Lambda$, Lebesgue) with $X$ b.c. at the boundary $\partial \Lambda$ of $\Lambda$, and $\mu \geqq 0$. Clearly

$$
V_{\Lambda, X}^{(\mu)} \leqq V_{\Lambda, X}^{\left(\mu^{\prime}\right)}, \text { for } \mu \geqq \mu^{\prime}>0 .
$$

Let $U_{\Lambda, X}^{(\mu)}\left(\xi^{n}, \bar{\xi}^{m}\right)$ be an $(n, m)$ particle Hamilton function defined as in (3.1), but with $V_{\Lambda, X}$ replaced by $V_{\Lambda, X}^{(\mu)}$ and with $W_{A, X}(\xi)$ replaced by $\frac{1}{2} \lim _{n \rightarrow \xi}\left(V_{\Lambda, X}^{(\mu)}(\xi, \eta)-V_{\infty}(\xi, \eta)\right)$. By (B.1)

$$
U_{\Lambda, X}^{(\mu)}\left(\xi^{n}, \bar{\xi}^{m}\right) \leqq \lim _{\mu^{\prime} \rightarrow 0} U_{\Lambda, X}^{\left(\mu^{\prime}\right)}\left(\xi^{n}, \bar{\xi}^{m}\right)=U_{A, X}\left(\xi^{n}, \bar{\xi}^{m}\right),
$$

and the last equation holds for all $X$, provided one sets $U_{A, X}\left(\xi^{n}, \bar{\xi}^{m}\right) \equiv+\infty$, for $n \neq m$, when $X=F, P$ or $N$. We define

$$
Q^{(\mu) X}(\Lambda, n, m, \beta)=\frac{1}{\mathrm{n} !} \cdot \frac{1}{m !} \int_{\Lambda^{n+m}} d \xi^{n} d \bar{\xi}^{m} e^{-\beta U_{\Lambda}^{(\mu)} X\left(\xi^{n}, \bar{\xi}^{m}\right)},
$$

and

By (B.2)

$$
\Xi^{(\mu) X}(\Lambda, z, \beta)=\sum_{n, m=0}^{\infty} z^{n+m} Q^{(\mu) X}(\Lambda, n, m, \beta) .
$$

$$
\left.\begin{array}{l}
Q^{X} \leqq Q^{(\mu) X} \\
\Xi^{X} \leqq \Xi^{(\mu) X}
\end{array}\right\} \text { for } \quad \mu \geqq 0
$$

i.e. it suffices to establish stability of a gas where the "vortices" interact through a Yukawa - rather than a Coulomb potential, and this will follow from an upper bound on $\Xi^{(\mu) X}$ of the form

$$
\Xi^{(\mu) X}(\Lambda, z, \beta) \leqq \exp [k(z, \beta)|\Lambda|],
$$

where $|\Lambda|$ is the area of $\Lambda$, and $k(z, \beta)$ is a finite constant, for all $z>0$ and all $\beta<4 \pi / R^{2}$. If $X=D, \bar{\Lambda}=\bigcup_{j=1}^{l} \bar{\Lambda}_{j}$, where $\Lambda_{1}, \ldots, \Lambda_{n}$ are disjoint, open sets, then

$$
U_{\Lambda, D}^{(\mu)}\left(\xi^{n}, \bar{\xi}^{m}\right) \geqq\left(\sum_{j=1}^{l} U_{\Lambda_{j}, D}^{(\mu)}\right)\left(\xi^{n}, \bar{\xi}^{m}\right),
$$

and one checks easily that this entails

$$
\Xi^{(\mu) D}(\Lambda, z, \beta) \leqq \prod_{j=1}^{l} \Xi^{(\mu) D}\left(\Lambda_{j}, z, \beta\right) .
$$

Thus it suffices to prove (B.6) for $X=D$ and a region $\Lambda$ of unit area. By (4.2), this will prove (B.6) for $X=F, P, N$, as well. In the literature one finds two fairly 
convenient methods for proving (B.6); see $[8,9,3]$. They both rely on the sineGordon transformation which also plays a crucial role in a proof of the monotonicity property (3.17) based on correlation inequalities [10]. We briefly recall some of the main formulas defining this transformation. Let $C\left(=V_{A, D}^{(\mu)}\right)$ be the integral kernel of a positive definite quadratic form on $L^{2}(\Lambda$, Lebesgue). Let $d \mu_{c}(\phi)$ denote the Gaussian measure on $\mathscr{D}^{\prime}(\Lambda)$ [the dual of $C_{0}^{\infty}(\Lambda)$ ] with mean 0 and covariance $C$. Let $C_{0}$ be a kernel with the property that

$$
c(\xi)=\lim _{\eta \rightarrow \xi}\left(C(\xi, \eta)-C_{0}(\xi, \eta)\right)
$$

is continuous and integrable near $\partial \Lambda$. Formally, we define random fields $: e^{i \alpha \phi}:_{0}$ and $: e^{i \alpha \phi}:$ by

$$
\begin{gathered}
: e^{i \alpha \phi}:_{0}(\xi)=e^{\frac{1}{2} \alpha^{2} C_{0}(\xi, \xi)} e^{i \alpha \phi(\xi)} \\
: e^{i \alpha \phi}:(\xi)=e^{\frac{1}{2} \alpha^{2} C(\xi, \xi)} e^{i \alpha \phi(\xi)} .
\end{gathered}
$$

It follows that

$$
: e^{i \alpha \phi}:_{0}(\xi)=e^{-\frac{1}{2} \alpha^{2} c(\xi)}: e^{i \alpha \phi}:(\xi) .
$$

By the definition of $d \mu_{C}$ and (B.8)-(B.10)

$$
\int \prod_{j=1}^{n}: e^{i \alpha_{j} \phi}:_{0}\left(\xi_{j}\right) d \mu_{C}(\phi)=\exp \left[-U\left(\xi_{1}, \ldots, \xi_{n}\right)\right],
$$

where

$$
U\left(\xi_{1}, \ldots, \xi_{n}\right)=\sum_{1 \leqq i<j \leqq n} \alpha_{i} \alpha_{j} C\left(\xi_{i}, \xi_{j}\right)+\frac{1}{2} \sum_{i=1}^{n} \alpha_{i}^{2} c\left(\xi_{i}\right) .
$$

If we set $C=V_{A, D}^{(\mu)}, C_{0}=V_{\infty}$ we obtain

\section{Lemma B.1.}

$$
\Xi^{(\mu) D}(\Lambda, z, \beta)=\left\langle e^{2 z \int_{\Lambda}: \cos \sqrt{\beta} R \phi: 0(\xi) d^{2} \xi}\right\rangle_{C},
$$

where $\langle(\cdot)\rangle_{C}$ denotes integration with respect to the Gaussian measure $d \mu_{C}(\phi)$, and $C=V_{\Lambda, D}^{(\mu)}, \mu \geqq 0$.

Remark. Formally, the proof follows by power series expansion of the exponential, the identity

$$
2 z: \cos \sqrt{\beta} R \phi:_{0}(\xi)=z\left(: e^{i \sqrt{\beta} R \phi}:_{0}(\xi)+: e^{-i \sqrt{\beta} R \phi}:_{0}(\xi)\right)
$$

and identity (B.11). To make these formal calculations rigorous, one first proves Lemma B.1 for a regularized version of $V_{\Lambda, D}^{(\mu)}(\xi, \eta)$ which is continuous in $\xi$ and $\eta$ and then removes the regularization, proving at the same time a uniform bound of the form (B.6). Details for $X=F$ may be found in $[8,9]$. For $X=D$, a convenient regularization consists in replacing

$$
V_{\Lambda, D}^{(\mu)}(\xi, \eta) \quad \text { by } \quad\left[V_{\Lambda, D}^{(\mu)}-V_{\Lambda, D}^{(M)}\right](\xi, \eta), \quad M \gg \mu>0 .
$$

This is used in [3]. 
By (B.10),

$$
\int_{A}: \cos \sqrt{\beta} R \phi:_{0}(\xi) d^{2} \xi=\int_{A} \exp \left[-\frac{\beta}{2} R^{2} c(\xi)\right]: \cos \sqrt{\beta} R \phi:(\xi) d^{2} \xi,
$$

where

$$
c(\xi)=\lim _{\eta \rightarrow \xi}\left[V_{\Lambda, D}^{(\mu)}(\xi, \eta)-V_{\infty}(\xi, \eta)\right],
$$

and we may choose $\mu=1$. One verifies easily that, for $\beta R^{2}<4 \pi$ and $\Lambda$ a unit square or unit disc

(a) $\int \exp \left[-\frac{\beta}{2} R^{2} c(\xi)\right] d^{2} \xi<\infty$, and

(b) $\int_{\theta \Lambda \times \theta \Lambda} \exp \left[-\frac{\beta}{2} R^{2}\{c(\xi)+c(\eta)\}\right] V_{\theta \Lambda, D}^{(\mu)}(\xi, \eta) d^{2} \xi d^{2} \eta \leqq$ const $\theta$,

for all $\theta \geqq 1$. To prove (a) and (b) we notice that $-c(\xi) \leqq(4 \pi)^{-1} \ln (1 / \operatorname{dist}(\xi, \partial \Lambda))$ + const and that $V_{\theta \Lambda, D}^{(\mu)}(\xi, \eta)$ has exponential decay in $|\xi-\eta|$ [this is used in the proof of (b)].

Estimates (a) and (b) are typical of the estimates one needs to control the renormalization group scheme in [3] which (with Lemma B.1) yields the bound

$$
\Xi^{(\mu) D}(\Lambda, z, \beta) \leqq \exp c\left(\beta R^{2}\right) z^{2},
$$

for some constant $c\left(\beta R^{2}\right)$ which is finite for $0<\beta R^{2}<4 \pi$, and $\Lambda$ is assumed to be a unit square or unit disc. The method in [3] is designed to establish (B.14) for a renormalized version of $\Xi^{(\mu) X}(\Lambda, z, \beta)$, for all $\beta R^{2} \leqq 6 \pi$. This causes some technical complications which are unnecessary in our case. It is not entirely trivial to develop a simplified version of [3] which can be used to prove (B.14) without appealing to sophisticated techniques. Since details are lengthy but fairly straightforward, we omit them.

When $\Lambda$ is a disc, one can set $\mu=0$ and prove (B.14) by following the method in $[8,9]$. This case is quite simple, because the Green's function of the Laplacian with Dirichlet b.c. on the boundary of a disc has a simple explicit expression. One proceeds as follows: One defines $C=V_{A, D}^{(0)}=V_{A, D}$. The Green's function $V_{A, D}$ is calculated in $[8,9]$. Let $\Lambda_{0}$ and $\Lambda$ be two discs centered at the origin, with $\Lambda_{0} \subset A$ and $\operatorname{dist}\left(\Lambda_{0}, \partial \Lambda\right)>0$. Then

$$
\begin{aligned}
\Xi^{D}(\Lambda, z, \beta)= & \left\langle\exp \left[2 z \int_{\Lambda}: \cos \sqrt{\beta} R \phi:_{0}(\xi) d^{2} \xi\right]\right\rangle_{C} \\
\leqq & \left\langle\exp \left[4 z \int_{\Lambda_{0}}: \cos \sqrt{\beta} R \phi:_{0}(\xi) d^{2} \xi\right]\right)_{C}^{1 / 2} \\
& \cdot\left\langle\exp \left[4 z \int_{\Lambda \sim \Lambda_{0}}: \cos \sqrt{\beta} R \phi:_{0}(\xi) d^{2} \xi\right]\right\rangle_{C}^{1 / 2}
\end{aligned}
$$

Now, notice that the re-Wick ordering factor $\exp \left[-\frac{\beta}{2} R^{2} c(\xi)\right]$ is uniformly bounded on $\Lambda_{0}$. Therefore a convergent upper bound on the first factor on the right side of (B.15) follows from the results in $[8,9]$. A bound for the second factor 
can be proven by adapting the arguments in Sect. 3 of [8]: In Eqs. (3.11) and (3.15) of [8] the terms $\prod_{j=1}^{n}\left|z_{j}-w_{j+n}\right|^{\alpha}\left|w_{j}-z_{j+n}\right|^{\alpha}$ are replaced by

$$
\prod_{j=1}^{n}\left|z_{j}-w_{j+n}\right|^{\alpha}\left|w_{j}-z_{j+n}\right|^{\alpha} \exp \left[-\frac{\beta}{2} R^{2}\left\{c\left(\left|z_{j}\right|\right)+c\left(\left|w_{j}\right|\right)\right\}\right],
$$

where $\alpha=\beta R^{2} / 4 \pi$, and

$$
-c(|z|)=\lim _{w \rightarrow z}\left[V_{\infty}-V_{\Lambda, D}\right](z, w) \leqq \frac{1}{2 \pi} \ln (1 / \operatorname{dist}(z, \partial \Lambda))+\text { const } .
$$

Since $\left|z_{j}-w_{j+n}\right| \leqq$ const $\operatorname{dist}\left(z_{j}, \partial \Lambda\right)$ and $\left|w_{j}-z_{j+n}\right| \leqq$ const $\operatorname{dist}\left(w_{j}, \partial \Lambda\right)$, for all $z_{1}, \ldots, z_{n}$ and $w_{1}, \ldots, w_{n}$ contained in $\Lambda \backslash \Lambda_{0}$, (B.16) is bounded by const ${ }^{n}$. The estimates in Sect. 3 of [8] and the boundedness of (B.16) yield a finite upper bound on the second factor in (B.15).

Finally, we note that the stability bound for $Q^{X}(\Lambda, n, m, \beta)$ follows from (B.14) and (B.4) by the Cauchy estimate.

We now outline the idea of the proof of the monotonicity properties (3.17) of the pressure. First note that

$$
\frac{\partial p_{\Lambda}^{X}(z, \beta)}{\partial z}=|\Lambda|^{-1} z^{-1} \int_{\Lambda} \varrho_{\Lambda}^{X}(\beta, z ; \xi) d^{2} \xi ;
$$

see (5.4). In the sine-Gordon representation

$$
\varrho_{\Lambda}^{X}(\beta, z ; \xi)=2 z \Xi^{X}(\Lambda, z, \beta)^{-1}\left\langle: \cos \sqrt{\beta} R \phi:_{0}(\xi) \exp \left[2 z_{\Lambda}: \cos \sqrt{\beta} R \phi:_{0}(\xi) d^{2} \xi\right]\right\rangle_{C},
$$

with $C=V_{A, X}$. It now follows from the correlation inequalities in [10] and the inequalities

$$
\begin{aligned}
& V_{\Lambda_{1}, N}+V_{\Lambda_{2}, N} \geqq V_{\Lambda, N}, \\
& V_{\Lambda_{1}, D}+V_{\Lambda_{2}, D} \leqq V_{\Lambda, D},
\end{aligned}
$$

where $\Lambda_{1}$ and $\Lambda_{2}$ are disjoint, open sets and $\bar{\Lambda}=\bar{\Lambda}_{1} \cup \bar{\Lambda}_{2}$, that for each fixed $\xi$

$$
\varrho_{\Lambda}^{X}(\beta, z ; \xi) \text { is increasing in } \Lambda \text {, for } X=F, N \text {, }
$$

and

$$
\varrho_{\Lambda}^{D}(\beta, z ; \xi) \text { is decreasing in } \Lambda .
$$

From this (3.17) follows by standard arguments (provided the domains are squares or discs).

We conclude with a few remarks on vortex gases with vortices, the vorticities of which are distributed according to a measure $d \lambda(R)$, with supp $\lambda \subset\left[-R_{0}, R_{0}\right]$, $R_{0}<\infty$. The grand partition function of this gas in the sine-Gordon representation is given by

$$
\Xi^{X}(\Lambda, \lambda, \beta)=\left\langle\exp \int d \lambda(R) \int_{\Lambda}: e^{i \sqrt{\beta} R \phi}:_{0}(\xi) d^{2} \xi\right\rangle_{C}
$$


$C=V_{A, X}$. By taking the absolute value of the expression inside the expectation $\langle(\cdot)\rangle_{C}$ we obtain

$$
\Xi^{X}(\Lambda, \lambda, \beta) \leqq\left\langle\exp \int d \lambda(R) \int_{\Lambda}: \cos \sqrt{\beta} R \phi:_{0}(\xi) d^{2} \xi\right\rangle_{C} .
$$

By Jensen's inequality

$$
\exp \int d \lambda(R) \int_{A}: \cos \sqrt{\beta} R \phi:_{0}(\xi) d^{2} \xi \leqq z^{-1} \int d \lambda(R) \exp \left[z \int_{A}: \cos \sqrt{\beta} R \phi:_{0}(\xi) d^{2} \xi\right]
$$

where $z \equiv \int d \lambda(R)$. Thus, combining (B.19)-(B.21) one finds

$$
\Xi^{X}(\Lambda, \lambda, \beta) \leqq z^{-1} \int d \lambda(R)\left\langle\exp z \int_{A}: \cos \sqrt{\beta} R \phi:_{0}(\xi) d^{2} \xi\right\rangle_{C} .
$$

The right side is bounded for all $z>0$ and uniformly in $R$, provided $\beta R_{0}^{2}<4 \pi$; see (B.14). If $d \lambda(R)=d \lambda(-R)$, the correlation inequalities in [10] are applicable, as well, and can be used to prove (3.17).

Acknowledgements. We thank O. E. Lanford III and T. Spencer for very helpful discussions.

\section{References}

1. Aref, H.: Motion of three vortices. Phys. Fluids 22, 393-400 (1979)

2. Aref, H., Pomphrey, N.: Integrable and chaotic motions of four vortices. Phys. Lett. 78A, 297-300 (1980)

3. Benfatto, G., Gallavotti, G., Nicolò, F.: On the massive sine-Gordon equation in the first few regions of collapse. Commun. Math. Phys. 83, 387-410 (1982); see also Benfatto, G., Cassandro, M., Gallavotti, G., Nicolò, F., Olivieri, E., Presutti, E., Scacciatelli, E.: Ultraviolet stability in Euclidean scalar field theories. Commun. Math. Phys. 71, 95 (1980)

4. Brydges, D.C.: A rigorous approach to Debye screening in dilute classical Coulomb systems. Commun. Math. Phys. 58, 313-350 (1978)

5. Brydges, D.C., Federbush, P.: Debye screening. Commun. Math. Phys. 73, 197-246 (1980)

6. Brydges, D.C., Fröhlich, J., Seiler, E.: On the construction of quantized gauge fields. III. The twodimensional Higgs model without cutoffs. Commun. Math. Phys. 79, 353-399 (1981)

7. Caillol, J.M. : Exact results of a two-dimensional one-component plasma on a sphere. J. Phys. Lett. 42, L-245-247 (1981)

8. Fröhlich, J.: Classical and quantum statistical mechanics in one and two dimensions, twocomponent Yukawa and Coulomb systems. Commun. Math. Phys. 47, 233-268 (1976)

9. Fröhlich, J.: Quantum sine-Gordon equation and quantum solitons in two space-time dimensions. In: Renormalization theory, pp. 371-414. Velo, G., Wightman, A.S. (eds.). Dordrecht, Boston: Reidel 1976

10. Fröhlich, J., Park, Y.M.: Correlation inequalities and the thermodynamic limit for classical and quantum continuous systems. Commun. Math. Phys. 59, 235-266 (1978)

11. Fröhlich, J., Spencer, T.: On the statistical mechanics of Coulomb and dipole gases. J. Stat. Phys. 24, 617-701 (1981)

12. Griffiths, R.B.: Free energy of interacting magnetic dipoles. Phys. Rev. 176, 655-659 (1968)

13. Gunson, J., Panta, L.S.: Two-dimensional neutral Coulomb gas. Commun. Math. Phys. 52, 295-304 (1977)

14. Jancovici, B.: Exact results for the two-dimensional one-component plasma. Phys. Rev. Lett. 46, 386-388 (1981)

15. Kraichnan, R.H., Montgomery, D.: Two-dimensional turbulence. Rep. Prog. Phys. 43, 547-619 (1980)

16. May, R.M.: Exact equation of state for a 2-dimensional plasma. Phys. Lett. 25A, 282 (1967) 
17. Montgomery, D., Joyce, G.: Statistical mechanics of "negative temperature" states. Phys. Fluids 17, $1139-1145$ (1974)

18. Novikov, E.A.: Dynamics and statistics of a system of vortices. Zh. Eksp. Teor. Fiz. 68, 1868-1882 (1975), English translation. Sov. Phys. - JETP 41, 937-943 (1975)

19. Novikov, E.A., Sedov, Yu.B. : Stochastic properties of a four-vortex system. Zh. Eksp. Teor. Fiz. 75, 868-876 (1978), English translation. Sov. Phys. - JETP 48, 440-444 (1978)

20. Onsager, L. : Statistical hydrodynamics. Suppl. Nuovo Cimento 6, 279-287 (1949)

21. Park, Y.M.: Massless quantum sine-Gordon equation in two space-time dimensions: correlation inequalities and infinite volume limit. J. Math. Phys. 18, 2423-2426 (1977)

22. Poincaré, H.: Théorie des tourbillons. Paris: Georges Carré 1893

23. Reed, M., Simon, B.: Methods of modern mathematical physics, Vol. II. Fourier analysis, selfadjointness. New York: Academic Press 1975

24. Riesz, F., Sz.-Nagy, B.: Leçons d'analyse fonctionelle (3e éd). Académie des Sciences de Hongrie 1955

25. Ruelle, D.: Statistical mechanics, rigorous results. New York: W. A. Benjamin 1969

26. Sari, R.R., Merlini, D.: On the $v$-dimensional one-component classical plasma: the thermodynamic limit problem revisited. J. Stat. Phys. 14, 91-100 (1976)

27. Sklyanin, E.K., Takhtadzhyan, L.A., Faddeev, L.D.: Quantum inverse problem method. I. Theor. Math. Phys. 40, 688-706 (1980)

28. Ziglin, S.L.: Non-integrability of a problem of the motion of four point vortices. Dokl. Akad. Nauk SSSR 250 No. 6, English translation. Sov. Math. Dokl. 21, 296-299 (1980)

Communicated by O. E. Lanford III

Received February 15, 1982 